NASA/TM-2001-211165

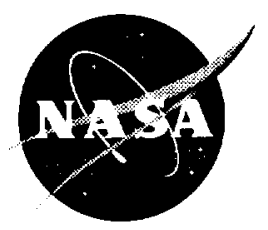

\title{
The Applicability of the Generalized Method of Cells for Analyzing Discontinuously Reinforced Composites
}

D.H. Pahr

Vienna University of Technology, Vienna, Austria

S.M. Arnold

Glenn Research Center, Cleveland, Ohio

National Aeronautics and

Space Administration

Glenn Research Center 


\section{Acknowledgments}

Scholarship funding for the first author to complete this work was provided by the "Akademisch-soziale Arbeitsgemeinschaft Österreichs (academic-social syndicate of Austria)."

\section{Available from}

NASA Center for Aerospace Information 7121 Standard Drive

Hanover, MD 21076
National Technical Information Service 5285 Port Royal Road Springfield, VA 22100

Available electronically at http://gltrs.grc.nasa.gov/GLTRS 


\title{
The Applicability of the Generalized Method of Cells for Analyzing Discontinuously Reinforced Composites
}

\author{
D.H. Pahr \\ Vienna University of Technology \\ A-1040 Vienna, Austria \\ and \\ S. M. Arnold \\ National Aeronautics and Space Administration \\ Glenn Research Center \\ Cleveland, Ohio 44135
}

\begin{abstract}
The paper begins with a short overview of the recent work done in the feld of discontinuous reinforced composites. focusing on the different parameters which influence the material behavior of discontinuous reinforced compasites. as well as the various analysis appraaches undertaken. Based on this overview it became evident. that in order to investigate the enumerated effects in an efficient and comprehensive manner, an alternative approach to the computationally intensive finite-element based micro-mechanics appraach is required. Therefore an investigation is conducted to demonstrate the utility of utilizing the generalized method of cells (GMC), a semi-analytical micromechanics-based approach. to simulate the elastic and elastoplastic material behavior of aligned short fiber composites. The results are compared with 1 ) simulations using other micromechanical based mean field models and finite element (FE) unit cell models found in the literature given elastic material behavior. as well as 2) finite element unit cell and a new semi-analytical elastoplastic shear lag model in the inelastic range. GMIC is shown to definitely have a window of applicability when simulating discontinuously reinforced compasite material behavior.
\end{abstract}

\section{Introduction}

Metal matrix compasites (MMCs) have received considerable attention over the past 30 years due to their attractive specific strength, stiffiness, fatigue and thermal properties[Clyne and Withers (1993)], [Kelly and Zweben (Eds) (2000)], [Sinclair and Gregson (1997)], [Lloyd (1994)]. MMIC materials can be generically classified by their type of reinforcement, which consists of either continuous fiber (identified herein as CFMMC) or discontinuous particulate (termed herein as DRX) reinforcements. While both classes of materials share common features at a fundamental level (e.g., matrix/reinforcement compatibility; interfacial properties sensitivity, architectural interaction (reinforcement size, shape and packing) and property optimization, at a practical level dramatic differences can and do exist. For instance. CFMMC ty pically have exceptionally high strength and stiffness capabilities that are inherently strongly anisotropic; whereas. DRX material properties are ty pically more isotropic in nature with lower specific strength, yet have excellent stiffness capability. Clearly, failure to appreciate and consider explicitly the appropriate reinforcement morpholog can result in inaccurate performance assessments, as elaborated upon in the next section. The combination of good transverse properties, relatively low cost. high workability and reasonable increases in 
performance over monolithic metallic alloys have made discontinuous reinforced materials the most commercially attractive system to date for many stiffness driven applications [Clyne and Withers (1993)], [Kelly and Zweben (Eds) (2000)], [Miracle and Maruyama (2000)].

Although manufacturing issues and cost are probably the greatest impediments to the wide application of these advanced materials, it is also clear that accurate design and life prediction tools are greatly needed to facilitate the implementation of these developing materials. Although closure has not been reached regarding the best models for use in design and life prediction, it has become evident that if a model is ever to serve a purpose beyond that of basic research, it must fulfill several primary requirements. These include a significant level of accuracy on both the macro and micro scales, computational efficiency; and compatibility with the finite element method. Fulfillment of these recuirements allows a model to serve the compasite developer by enabling quick and easy variation of composite parameters for material development and optimization purposes. Likewise, thase who attempt to utilize composites in structural design are well served if the model is compatible with the finite element method. The generalized method of cells (GMIC), originally developed by Aboudi (1991, 1995), is a good choice for implementation into modeling tools for advanced composites. given the requirements described above. Particularly; whenever microfield as well as macrofield quantities will be needed - as is the case when attempting to acoount for inelastic material behavior in MMIS.

GMIC is a fully analytical micromechanics model for multi-phased materials with arbitrary periodic microstructures. It provides pseudo closed-form multiaxial constitutive equations for such materials, and allows straightforward implementation of physically-based viscoplastic deformation models, as well as arbitrary failure and damage models for each phase. Further, recent independent advanoes have simplified the implementation of GMC as an elemental constituent material model in finite element analysis [Arnold et al, (1999)], and significantly increased the model's computational efficiency [Pindera and Bednarcyk (1999)]. GMC has been implemented in the NASA Glenn Research Center oomprehensive micromechanics analysis code, MAC/GMC [Amold et al, (1999)]. The code has many features that render it useful for design, deformation modeling, and life prediction for a wide range of materials. These features include the ability to simulate general thermomechanical loading on compasites whase geometries are represented by a library of continuous and discontinuous repeating unit cells, a library of nonisothermal elastic/viscoplastic constitutive models, fatigue damage analysis, yield surface analysis, laminate analysis, and interface modeling.

In the past, the various capabilities described above have been exercised extensively, however, the predominant material system examined has been that of the CFMMC [Aboudi(1996)], [Arnold, et. al. (1996)], [Goldberg and Arnold (2000)], [Iyer et al. (2000)], [Bednarcy'k and Amold (2000a)], [Lissenden et al. (2000)], [Bednarcyk and Arnold (2000b)]. Consequently; the primary objective of this current study is to assess the applicability of GMC in predicting the elastic and inelastic (plastic) behavior of particulate reinforced composites (DRXs). The literature indicates that DRX behavior is significantly influenced by various characteristic parameters like fiber arrangements (staggered, non-staggered, cluster, random, packing type), fiber volume fraction, fiber aspect ratias, fiber-matrix stiffiness ratios and reinforcement type (cylinder, particle, etc. ). A brief review of this is provicled in the background section. Even though GMC is fully capable of modeling arbitrary multiphased composite architectures, to facilitate comparison with previous work reported in the literature and limit the scope of this investigation, only one type of DRX is considered, namely, the simulation of aligned short fiber compasites with non-staggered cylindrical reinforcements and a hexagonal packing arrangement. The need for such an assessment stems from the fact that although GMC consists of analytical equations that are able to deal with both the homogenization and localization process for composite materials in a very numerically efficient way, the method's linear displacement field assumption in combination with the imposition of displacement and traction boundary conditions in an average sense, gives rise to an inherent lack of normal and shear component coupling. This lack of coupling is such that application of a global normal (or shear) stress field will produce only a local normal (or shear) stress field. Consequently, for cases (e.g., DRX materials) in which the load transfer mechanism between phases (e.g., matrix and fibers) is shear dominated, the lack of normal-shear coupling may become quite problematic. In particular, in the case of an elastoplastic matrix regime placed between two short fibers, GMC's lack of shear coupling recuires both the fiber and matrix to carry the same normal stress component. This tends to produce an overly compliant (soft) composite response. The results presented within demonstrate this 
fact, and more importantly characterize the window of applicability for the GMC method within the context of discontinuously reinforced metallic composites. The introduction of an "artificial" elastoplastic matrix material between the particulate reinforcements, is propased in an attempt to extend the applicability of GMIC. Although, providing more reasonable macro behavior, such a strateg. still results in incorrect micromechanical stress fields within the unit cells, thereby liniting the usefulness of the current GNIC model when calculating failure or fatigue analyses.

With section 2, the paper begins by reviewing recent work done in the field of short fiber composites so as to reveal the key parameters influencing DRX material behavior. Section 3 then articulates the various analytical and numerical approaches employed and compared in this paper. Finally, section 4 presents a comparison of GMIC results with other analytical and numerical results within the elastic and elastoplastic range, respectively.

\section{Background: Factors Influencing DRX Materials}

The early work on the principles of strengthening in composites reinforced with discontinuous fibers, whiskers or particulates carried out in the $1950^{\circ}$ s and 1960 's is characterized by the use of relatively simple models that do not explicitly take into acoount the actual inclusion distribution through interaction between adjacent inclusions. Many models have been employed to study the effect of inclusion shape on the elastic moduli, as well as subsequent inelastic response, and also the mechanism of stress transfer from the matrix to a finite-length fiber. For instance, Eshelby's analytical solution to the problem of an arbitrarily shaped ellipsoidal inclusion embedded in an homogeneous material deformed by uniform tractions or displacements at infinity has formed the basis for calculating the effective response of macrosoopically homogeneous twophase composites using a number of approaches [Eshelby (1957)]. These approaches include the self-oonsistent scheme which neglects the inclusion/matrix interaction in calculating stress fields in the inclusion phase [Hill (1965)], and the Mori-Tanaka method (NTM) which takes this interaction into account in an approximate fashion [Mori and Tanaka (1973)]. The problem of an array of ellipsoidal inclusions with different aspect ratios embedded in an elastoplastic matrix can be treated using the Mori-Tanaka approach, as was done by [Brown and Clarke (1975)] investigating the effect of inclusion shape on work hardening of metal matrix composites. However, this method ty pically underestimates yielding and subsequent hardening effects due to the use of mean stress and strain fields in the matrix phase, and treats all inclusion distributions on the same footing so long as macrosoopic homogeneity is preserved. Along similar lines, the so-called shear-lag analysis has been employed to study the effectiveness of short fibers as reinforcement using the strength-of-materials approach to analyze stress fields around and within a finite-length fiber embedded in a surrounding matrix [Dow (1963)]. While this type of analysis helps to identify shearing of the matrix as the primary mechanism of force transfer from the matrix to the fiber, and thus the critical fiber length over which the axial stress is introduced into the fiber from both ends, it is based on a very simplified one-dimensional analysis of stress fields which neglects the influence of morphologr of surrounding fibers, among other things.

Despite the relatively long history of modeling the response of discontinuous fiber compasites, only recently have systematic investigations of the effects of reinforcement shape and arrangement been initiated for these types of compasites. Inclusion of the thind dimension in the analysis of the elastic and inelastic response of discontinuous fiber composites increases the number of variables several fold relative to the analysis of continuous fiber composites. Thus, in addition to the arrangement and shape of the reinforcement in the plane transverse to the loading direction, variables associated with the planes parallel to the loading direction were included. These variables include the fiber aspect ratio, fiber spacing in the vertical and horizontal directions, thase associated with the effect of fiber clustering, and the extent of overlap between adjacent columns of fibers. These added complexities ty pically recuire numerical solution procedures, such as the finite-element analysis; particularly if complicated reinforcement shapes and arrangements are involved.

Finite-ement investigations carried out in recent years have focused on separating the effects of inclusion shape from the effects of inclusion packing array geometry on the overall elastic, elastoplastic and creep behavior. Due to many different ty pes of reinforcement employed in discontinuously-reinforced composites, including whiskers, particulates, platelets as well as finite-length fibers, extensive analyses of inclusion shape 
have been conducted for this class of composites. Examples of inclusion shapes investigated include spherical particles, circular cylinders with different aspect ratios, truncated circular (octagonal) cylinders, double-cone particles, and ellipsoidal or disk-like particles(platelets). Similarly, different packing arrays studied include hexagonal arrangements of inclusions in the plane of loading distributed in either regular (aligned) arrays or off-set (staggered) arrays in the planes parallel and perpendicular to the loading direction so as to model and assess the influence of distribution randomness that is typical in DRX composites. Inclusions such as disklike particles arranged in packet morphologies have also been considered, as have clustered arrangements. These investigations have been driven in large part by the wide range of inclusion morphologies that result from current material processing techniques, as well as by the wide range of shapes available for the inclusion phase.

In order to reduce the complexity of a threedimensional finite-element formulation in analyzing the response of discontinuous fiber, whisker or particulate composites, various idealizations of the unit cell have been employed by a number of researchers (e.g. clusters of whiskers or cylindrical particles in the plane parallel to the applied load have been modeled using a plane strain idealization of rectangular platelets). This effectively reduces the problem to that of a continuously reinforced composite subjected to loading in the plane perpendicular to the long fiber direction. The results of such analysis for rectangular crosssections with different aspect ratios in the plane of loading should be easily deduced from the early analysis of continuously reinforoed compasites with rectangularly shaped fibers [Ashton et al.(1969)]. In the case of a hexagonal array of inclusions in the plane transverse to the loading direction, the problem is often rectuced to an axisymmetric (i.e., two-dimensional) problem by approximating the unit cell using a circular cylinder with different types of lateral boundary conditions to simulate the interaction with adjacent fibers (i.e., unit cells). Using this model, different fiber arrangements in the plane parallel to the applied load have been investigated, with varying amounts of overlap between vertical columns of adjacent fibers (e.g. [Christman et al. (1989)], [Tvergaard (1990)], [Dragone and Nix (1960)], [Povirk et al. (1990)], [Yang et al. (1991)], [Bao et al.(1991)], [Siegmund et al. (1992)]). In contrast to continuously reinforoed composites, the inclusion arrangement in discontinuously reinforced compasites is inherently threedimensional, so that the use of plane models requires considerable caution. The results of fully three-dimensional finite-ement analyses of such periodic arrays are also available (e.g. [Levy and Papazian (1990)], [Weissenbek and Rammerstorfer (1993)], [A bel et al.(1993)], [Weissenbek et al. (1993)]). They are, however rather restrictive in terms of fiber arrangements and shapes, and tend to entail considerable computational costs. Both threedimensional and axisymmetric unit cell models have been used successfully for studying the nonlinear thermomechanical behavior of aligned short reinforced compasites.

The literature survey presented indicates significant influence of both inclusion shape and distribution on the elastic, elastoplastic and creep response of discontinuous fiber composites. The extent of this influence for a given fiber architecture depends on the inclusion content, inclusion/matrix material property mismatch, and the direction of applied load with respect to the internal micro-structure. The influence of fiber architecture on the response of DRX composites is significant and is based on a substantial number of investigations, each of which was necessarily limited in soope due to the prevalent use of the finite-element approach in modeling the response of the investigated architectures. Thus while a considerable body of knowledge has been generated that sheds light on the effectiveness of different fiber architectures in strengthening discontinuous fiber composites, considerably more systematic research is required to develop design guidelines for optimization of material performance through fiber anchitecture manipulation.

However, it becomes clear, based on the above discussion, that in order to investigate the enumerated effects in an efficient and comprehensive manner, including parametric studies involving fiber content and material property mismatch variations, an alternative approach to the computationally intensive finiteelement based micromechanics approach is required. This is particularly true when inelastic (viscoplastic) theories, which typically require computationally intensive integration algorithms, are employed to model the response of metallic matrices. Therefore, the objective of this paper is to apply the computationally efficient GMIC method so as to determine its suitability for predicting the macrascopic response of aligned, DRX materials. 


\section{Modeling Approaches}

All simulations conducted in this study consist of aligned short fiber composites, wherein the particulates (wiskers) are idealized as non-staggered. cylindrical reinforcements, with a hexagonal packing arrangement. Similarly other basic constraints imposed are as follows:

- The inclusion properties are assumed isotropic and linear elastic, while the matrix is taken to be isotropic elastoplastic with isotropic hardening. The material data for both the inclusion and matrix are treated as temperature-inclependent and isotropic.

- The inclusions are axisymmetric, identical in shape and size and can be characterized by an aspect ratio, $a_{r}$, that relate the length of an inclusion to its diameter.

- Investigated model parameters are the fiber (inclusion) volume fraction $\left(V_{f}=0.2 .0 .5\right)$, the fiber-matrix stiffiness ratio $\left(s_{r}=E_{F} / E_{M}=3.30\right)$ and the fiber aspect ratio $\left(a_{r}=l / d=5.15 .25\right)$.

- The inclusions and matrix are well bonded at their interface and remain that way during deformation. Thus, we do not consider interfacial slip, fiber-matrix debonding or matrix micro-cracking.

- No time dependence (relaxation or creep) effects are included in the present analysis.

Note, obtaining experimental reference data for unidirectional short fiber composites appears to be problematic, as it has not proved passible to produce physical samples with perfectly aligned fibers. Consequently; in this paper we avoid this additional complexity of orientation effect by using threedimensional finite element analysis of aligned short fiber compasites as our reference standard, rather than experimental results.

\subsection{Material Data}

The material properties used for all calculations are given in Table 1. These properties are in the range of typical fiber-reinforced engineering thermo-plastics (PMC's - Material 1) and metal matrix compasites (NIMIC's - Material 2), respectively: The following input data are needed for both components: Young's modulus $E$ and Poisson's ratio $\nu$. The yield stress $\sigma_{Y}$ and the hardening modulus $E_{H}$ are needed for the matrix material only:

Table 1: Prototypical material properties used for all calculations.

\begin{tabular}{|c|c|c|c|c|}
\hline & \multicolumn{2}{|c|}{ Material 1 $\left(s_{R}=30\right)$} & \multicolumn{2}{c|}{ Material 2 $\left(s_{K}=3\right)$} \\
\hline Property & Fiber & Matrix & Fiber & Matrix \\
\hline$E(\mathrm{GPa})$ & 300. & 10. & 300. & 100. \\
\hline$\nu$ & 0.17 & 0.33 & 0.17 & 0.33 \\
\hline$\sigma_{Y}(\mathrm{MPa})$ & - & 20. & - & 200. \\
\hline$E_{H}(\mathrm{GPa})$ & - & 0.1 & - & 1.0 \\
\hline
\end{tabular}

\subsection{Analytical Models}

In the past, numerous analytical models have been examined in an attempt to determine the "best" (see e.g., [Tucker and Liang (1999)], [Aboudi (1991)]) available analy tical model for predicting the elastic stiffness behavior of aligned short fiber reinforced composites relative to reference FEA-model representations. Analytical models considered, were models such as: the Mori-Tanaka method (MTM)] [Mori and 


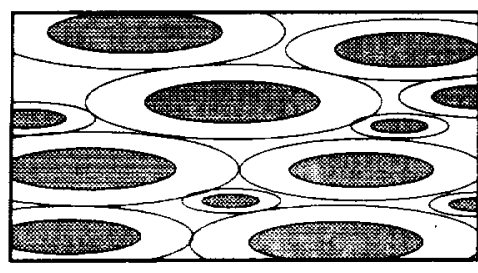

(a)

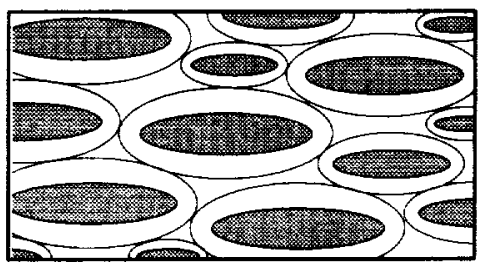

(b)

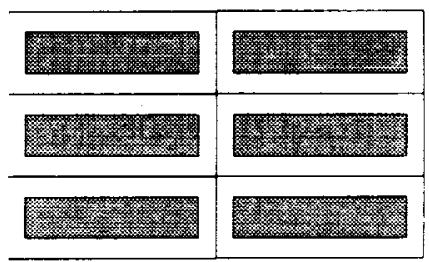

(c)

Figure 1: Sketch of inclusions-matrix shapes for the applied aligned short fiber compasite models. (a) MoriTanata model, (b) Hashin-Shtrikman estimates of Ponte Castañeda and Willis, (c) GMIC-model ( $\left.a_{r}=5.0\right)$

Tanaka (1973)], self-consistent models, bounding models (e.g., variable constraint model (VCNI) and HashinShtrikman bounds and estimates), Halpin-Tsai equations and shear lag models (see Appendix $B$ for details of the elastoplastic extension used in this study). For example, the recent work of [Tucker and Liang (1999)] recommended that the Mori-Tanaka model was the "best" choice for estimating the overall elastic stiffness behavior of aligned short fiber compasites.

In this study we will extend the scope of comparison to include the semi-analytical generalized method of cells model (GMIC) and compare GMC with the MTTM. VCM, FEM results and Hashin-Shtrikman estimates (HSE) $^{1}$ of [Ponte Castañeda and Willis (1995)]. Benveniste (1987) has provided a particularly simple and clear explanation of the Mori-Tanaka approach. Analytical bounds for the stiffiness behavior can be obtained from the variable constraint model (VCM), published by- [Pedersen and Withers (1992)]. From a practical point of view it is of interest that the lower (VCM) bound corresponds to the standand Mori-Tanaka results, while the upper (VCMI) bound can also be obtained from the Mori-Tanaka approach after a so called "color inversion" (i.e., exchanging the roles of inclusion and matrix). These bounds obtained from the Mori-Tanaka approach also correspond to the Willis bounds in the case of aligned inclusions, compare for example, [Weng (1990)].

It is important to remember that the basic assumption of the Mlori-Tanaka method is that the aligned ellipsoidal inclusions are surrounded by coaxial aligned ellipsoidal matrix domains in which both ellipsoids have the same aspect ratio (Fig.1a) and that each inclusion (fiber) within a concentrated composite sees the average strain of the matrix. Consequently, the MTM provides only macro (average) fields and properties and therefore lacks the ability to address adequately problems dominated by microfield quantities, such as damage and inelastic behavior analysis. Alternatively, GMC allows both homogenization and localization of the associated stress and strain fields (see Appendix A), thereby providing not only macro stiffness properties but also local eigenstrain field quantities. Note, that in the present investigation the GMIC unit cell differs from MTMI as different fiber-unit cell aspect ratios (Figs.2 and 3) can be realized since the whole inclusion (fiber) is surrounded by a matrix material layer of constant thickness (side-to-side and end-to-end distances are equal) and labeled, $e$, subsequently. Because of this varying aspect ratio, HSE for the prediction of the effective moduli of the elastic inhomogeneous material given in [Ponte Castañeda and Willis (1995)] are also introduced (Fig. 1b) to overcome the drawback of the original Mlori-Tanaka method so that different fiber-unit cell aspect ratios can be realized. Note, these Hashin-Shtrikman estimates correspond identically with the Mori-Tanaka method for the case of aligned, coaxial inclusions with equal aspect ratios of the inclusion and the surrounding matrix ellipsoids (see [Hu and Weng (2000)])

\footnotetext{
${ }^{1}$ Note. the Hashin-Shtrikman estimates differ from the Hashin-Shtrikman bounds. as [Ponte Castañeda and Willis (1995)] and [Willis (1977)] removed the restriction of statistically isotropic overall material symmetry (used by both HS and Willis) by separating the spatial distribution of inclusions from the inclusion shape. Also in the HSE. [Ponte Castañeda and Willis (1995)] employed only a single reference material (e.g.. the effective material like in self consistent models) instead of the two used by [Willis (1977)].
} 


\subsection{Generalized Method of Cells}

Although, GNIC is capable of analyzing any multiphased compasite material with an arbitrary internal microstructure and reinforcement shape (see Appendix A), the unit cell analyzed in this study is shown in Fig. 2. Due to the periodic boundary conditions inherent to the GNIC-model the whole RVE must be modeled. instead of only a quarter of the unit cell (see the dashed rectangle in Fig. 2) as typically done in the case of FEA representations where symmetric boundary conditions are applied. Apart from this

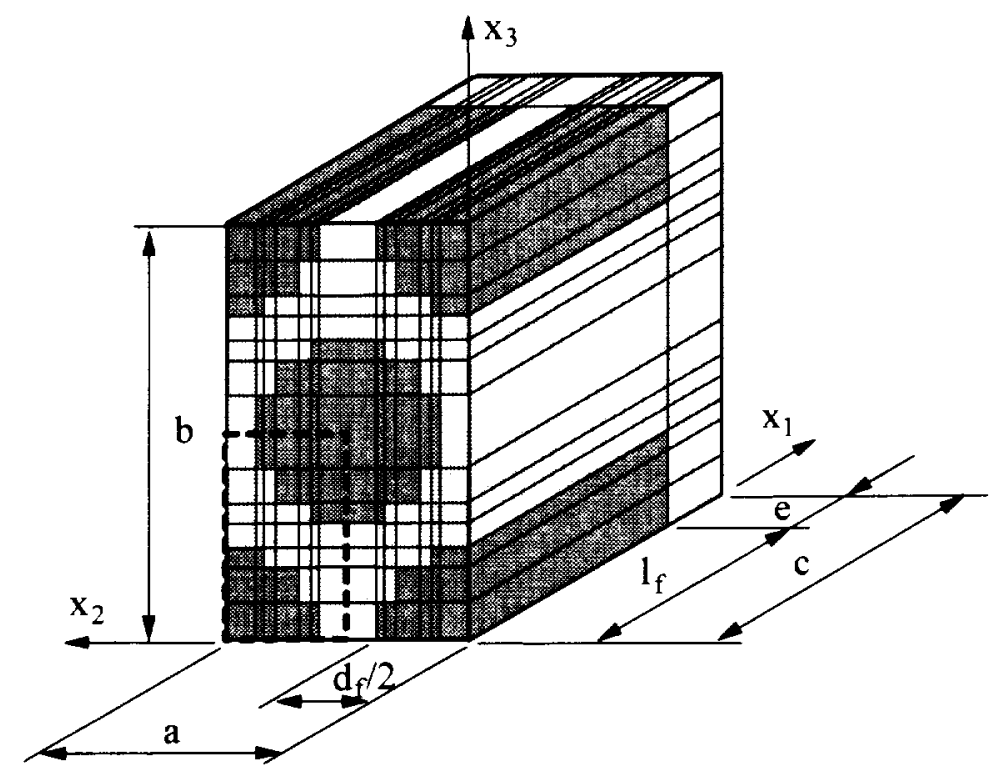

Figure 2: Aligned short fiber GNIC unit cell model, comprised of 338 subcells.

fact, the unit cell dimensions were chosen similar to the dimensions of the FE-model (see Fig. 3). The fiber was approximated with more than one subcell (sufficient for the longitudinal stiffness behavior) in order to reduce the error in the transverse response (i.e., the difference between $E_{22}$ and $E_{33}$ ) and better approximate the targeted transversely isotropic response behavior resulting from a hexagonal packing arrangement. The crass sectional area of the approximated fiber is taken equal to the crass sectional area of the actual circular fiber. These conditions, together with the known fiber diameter, fiber volume fraction, and the distance, $e$ (see Fig. 3) suffice to determine the dimensions of the unit cell for each fiber aspect ratio $a_{r}=l_{f} / d_{f}$. All GMC analyses were realized with the computer code MAC/GNC [Arnold et al.(1999)] developed at the NASA Glenn Research Center.

\subsection{Finite Element Models}

The finite element unit cell analyzed consisted of an identical periodic, three dimensional non-staggered array of fibers (similar to that idealized with GMIC) with the corresponding dimensions shown in Fig. 3. The unit cell dimension were chosen such that $b$ would be proportional to $a$ (i.e.. $b=3 a$ ) through the constant 3. In the case of hexagonal packing, $3=\sqrt{3}$, and the distance between neighboring fiber ends (end-to-end distance, equal to $c-l_{f}$ in Fig. 3) was set to the closest distance between two fibers (side-to-side distance. equal to $e$ in Fig. 3).

These conditions, together with the fiber diameter and volume fraction, suffice to determine the dimension $a . b$ and $c$ for the repeating volume element (RVE) to be analyzed. Note that a new unit cell and its corresponding 3D mesh are generated for each fiber aspect ratio $(l / d)$ and fiber volume fraction $\left(v_{f}\right)$. 
GMC unit cell

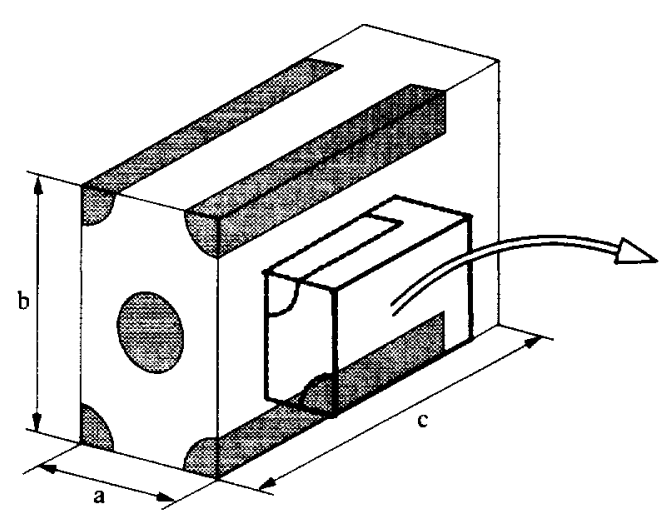

FE unit cell

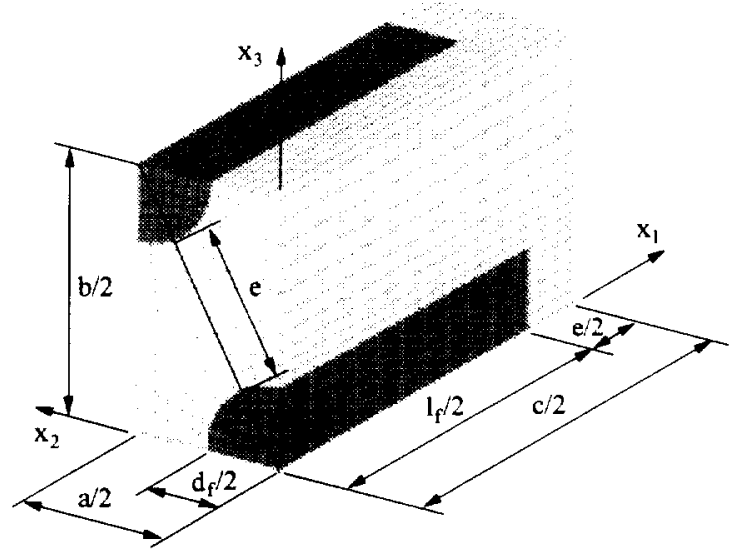

Figure 3: Example representative volume element and finite element unit cell for $a_{k}=5.0$ and $V_{F}=0.2$

Stiffnesses within the elastic and plastic range of these unit cells were calculated using [ABAQUS (1998)]. Fully integrated, first order, solid elements ${ }^{2}$ were used, as this element ty pe does not suffer from volumetric locking, which is critical for accurate elastoplastic calculations. The mesh discretization containing 13,300 elements employed is shown in Fig. 3. This mesh density is approximately three times finer than that used by [Weissenbek and Rammerstorfer (1993)] and was shown during the course of this work (by convergency. studies) to be sufficiently accurate for both elastic and inelastic analysis. Modeling of only one eighth of the whole RVE (depicted on the left) was achieved due to the use of appropriate symmetric boundary conditions. For example, when considering axial or transverse loading, sjmmetry requires all faces of the unit cll to remain plane. Consequently; to determine $E_{11}$ and $\nu_{12}$ the normal displacements of the front $\left(\mathrm{x}_{1}=0\right)$, left $\left(x_{2}=a / 2\right)$ and bottom $\left(x_{3}=0\right)$ faces of the unit cell were fixed. Then a master node was defined (the intersection point of the back, right and top face) and linear constraint equations applied on the back, right and top face such that the normal displacements of all nodes (except the master node) on these faces were set equal to the normal displacements of the master node. The tangential displacements on all faces were left unconstrained. The average (maroo) stress was then computed from the reaction force of the master node divided by the cross-sectional area normal to the reaction force of the investigated unit cell. Similarly; the average (macro) strain was computed by dividing the displacement of the master node by the corresponding length of the unit cell. For convenience, a one Newton load was applied to the unit cell.

Analogous conditions were used to load the unit cell in the $x_{2}$-direction to determine $E_{22}$ and $\nu_{23}$. The fifth and final independent material parameter for transversal isotropic material, the longitudinal shear modulus $G_{12}$, could also be determined using the unit cell shown in Fig. 3, assuming the appropriate periodic boundary conditions were applied to each face. This more complicated analysis was not undertaken at this time.

\section{Results}

\subsection{Elastic Material Behavior}

All of the analytical micromechanics theories used in this study predict full transversely isotropic properties. Transverse isotropy about the $x_{1}$ axis implies that the stiffiness behavior is the same for any loading

\footnotetext{
${ }^{2}$ In [ABAQUS (1998)] this is the element known as C3D8.
} 
direction in the 2-3 plane; consequently; this not only requires that $E_{22}=E_{33}$, but also that

$$
G_{23}=\frac{E_{22}}{2\left(1+\nu_{23}\right)}
$$

Therefore, only five independent stiffiness constants (e.g., $E_{11}, E_{22} . \nu_{12} . \nu_{23}$ and $G_{12}$ ) need be determined for the case of transverse isotropy. Periodic unit cells with hexagonal packing should be transversely isotropic as there are six planes of symmetry. However, since periodic boundary conditions were not applied to the FE unit cell. we were unable to obtain $G_{12}$ from the FE investigations. Consecquently; we will limit our comparison for the rarious approaches to the prediction of $E_{11}, E_{22}, \nu_{12}$ and $\nu_{23}$ only:

Results of the normalized axial modulus ( $x_{1}$-direction, scaled by the matrix modulus) obtained from the VCNI-model (NITMI-model), Hashin-Shtrikman estimates, GNIC-, and FE-model are shown in Fig. 4 (DRX like materials with a lower stiffness ratio, e.g.. $s_{r}=3$ ) and Fig. 5 (PMC like material, with a higher stiffness ratio, e.g. $\left.s_{r}=30\right)$ for numerous fiber aspect ratios and two fiber volume fractions, a low $\left(v_{f}=0.2\right)$ and a high $\left(v_{f}=0.5\right)$. The Hashin-Shtrikman-, GNIC- and FE-models were evaluated only at discrete aspect ratios: whereas, the VCM-result were computed for many different aspect ratios and thus displayed with a solid line. Note, for high stiffness ratios only the lower VCM-bound (which corresponds to the MTMI) was plotted. From Figs. $t$ and 5 it is apparent that the influence of fiber aspect ratio on $E_{11}$ is significant (Max. error of $35 \%$ ) for a composite with a high stiffness ratio whereas relatively insignificant (Max. error of $3 \%$ ) for materials with a low stiffiness ratio. In both cases the Hashin-Shtrikman estimate lay as expected within the upper and lower VCMI bounds. The GMIC- and FE-results are partially out of the Mori-Tanka based VCM-bounds due to the different inclusion topology (ellipsoidal versus cylindrical). Cenerally speaking. the GMC-model underestimates the longitudinal stiffness, particularly for high stiffiness ratios, although the trend is captured quite nicely: Alternatively, for materials with low stiffness ratios. good agreement between GMC and Hashin-Shtrikman estimates as compared with FE-results are observed given a wide range of aspect ratios and fiber volume fractions.

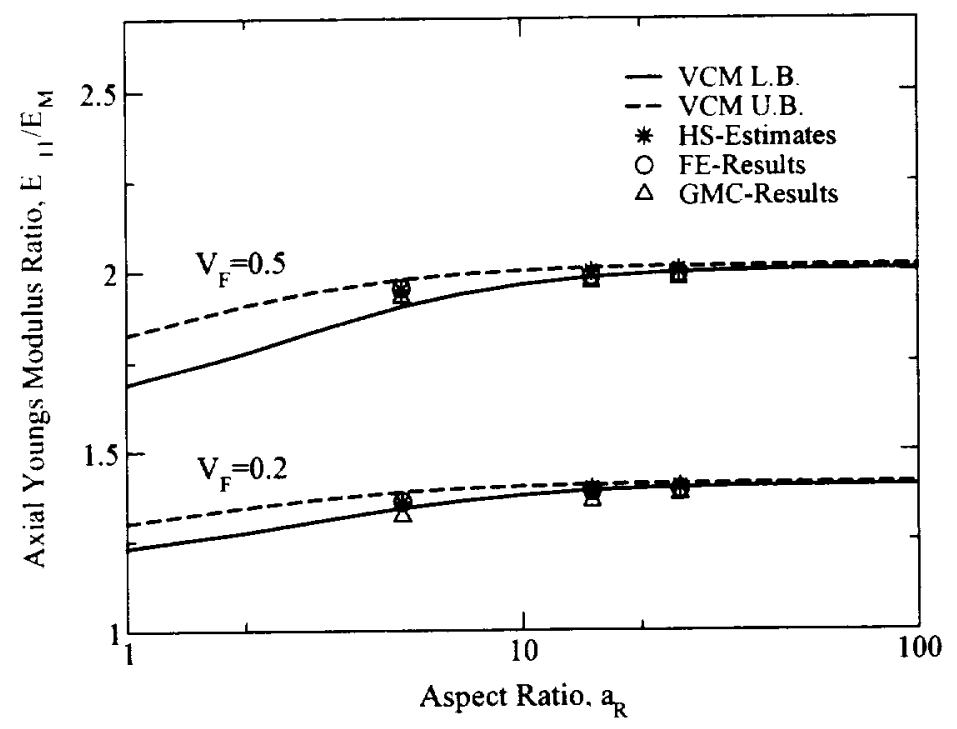

Figure 4: Comparison of axial modulus ratios for $s_{r}=\frac{E_{E}}{E_{M}}=3$

The physical reason for the difference between the FEA-results and those of GNIC can be inferred from Fig. 6, where the longitudinal stress distributions for a load in the $x_{1}$-direction are shown. In both FEA analyses shown in Fig. 6, the geometry; boundary conditions, and applied foroes are the same. Only the stiffness ratio between matrix and fiber constituent material is different. Evidently; these stress contours 


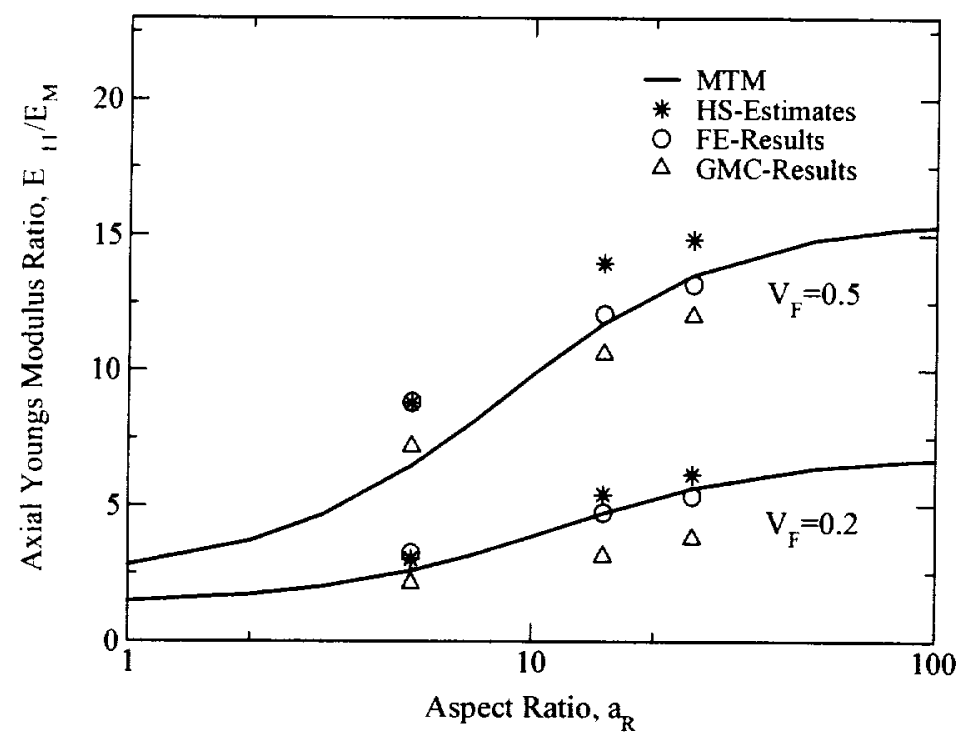

Figure 5: Comparison of axial modulus ratios for $s_{r}=\frac{E_{E}}{E_{M}}=30$

indicate that an increase in the stiffness ratio leads to a decrease in the matrix loading and conversely to an increase in the fiber loading (see maximum and minimum stress values). Similarly, the difference of the "mean" matrix stress in the volume of material between the two fiber ends as compared with the "mean" fiber stress becomes greater as the stiffness ratio is increased. Considering, the GMC representation, the stresses are constant and equal along a longitudinal fiber-matrix row. Therefore the error for lower stiffness ratios is decreased, yet when the stiffness ratio increases so too does the error. Figure 6 also gives an explanation as too why the GMC stiffness results are slightly under the FE-results in Fig. 4, as the GMC assumptions lead to an unloading of the "stiff" fiber and to an additional loading of the "soft" matrix.

Figures 7 and 8 illustrate the normalized transverse modulus results obtained from the various analytical and numerical methods considered. Here as one would expect, the fiber aspect ratio has little, if any; effect on the transverse modulus Also, in general the GMC results compare favorably (maximum error of approximately $5 \%$ ) with the FE-results throughout. For both stiffness ratios, the Hashin-Shtrikman estimates are equal to the lower VCM bound (which in turn are equal to the original MTM-results). Noteworthy is the fact that the GMC-results are much claser to the FE-results for the case of high stiffness ratios, low aspect ratios, and high fiber volume fractions than the other analytical estimates. Similarly, as observed for the longitudinal stiffiness the qualitative trend of the FE results is better captured by. GMC.

To help explain this observation, we examine the calculated transverse stress distribution obtained from FEA, as shown in Fig. 9. Note, only the case for the low stiffness ratio $\left(s_{r}=3\right)$ is shown in Fig. 9 as the FE-results for the higher stiffness ratios appear similar. Relative to the FE-results for the longitudinal loading case, the difference in the "mean" matrix stress of the volume of material between the two fibers in the loading direction and that of the "mean" fiber stresses for the transverse loading case are small. Consequently, the inaccuracy caused by having similar stress states in a given row or column of subcells within GMC is small; thus explaining the lack of influence the stiffness ratio has on the GMC predictions relative to thase determined using an FEA unit cell.

Figures 10 and 11 show a comparison of the axial Poisson's ratios based on the various evaluation methods. Again the comparison between FEA and GMC results are quite favorable, irrespective of aspect ratio and stiffness ratio; the difference being approximately a maximum of $5.2 \%$. Again, the GMC results follow the qualitative trend of the FE-results much better than either the Hashin Shtrikman estimates or MTM-results, especially for the case of high stiffness ratios and low fiber aspect ratios. 

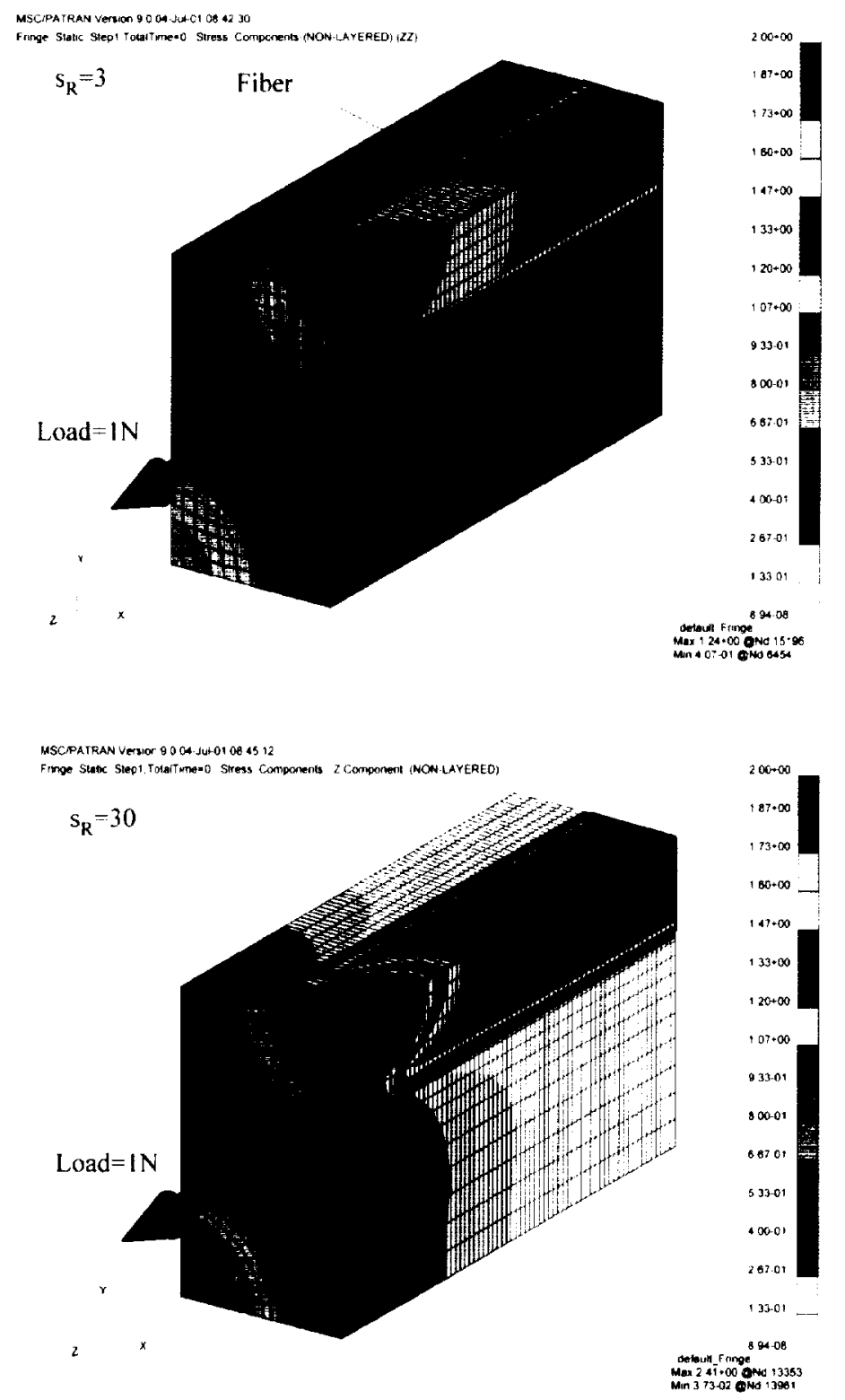

Figure 6: Example for the longitudinal stresses obtained from FE-calculation for $s_{r}=3$ and $s_{r}=30$ $\left(V_{F}=0.2 . a_{R}=5.0\right)$ 


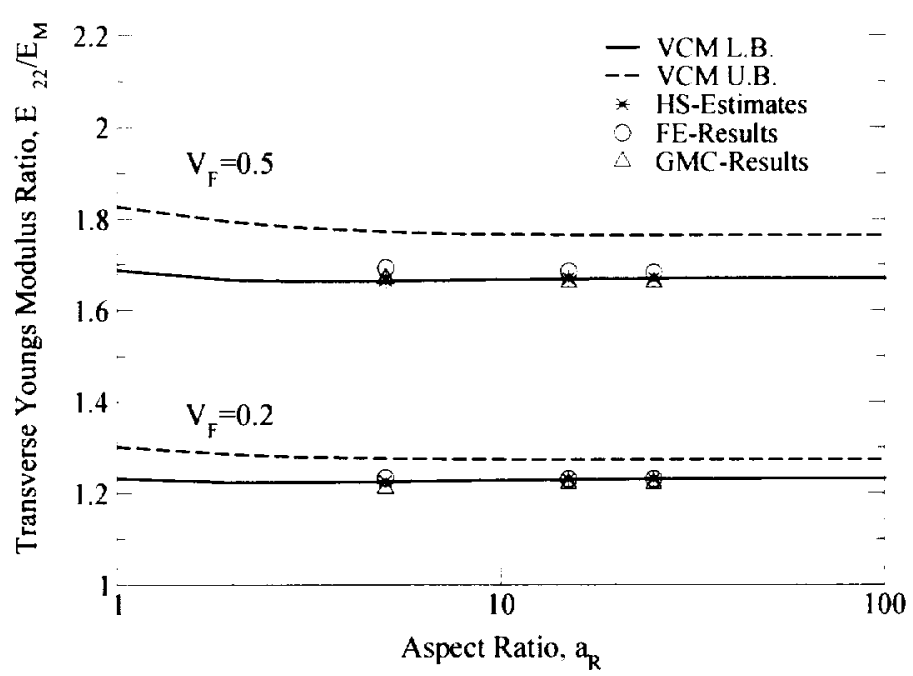

Figure 7: Comparison of transverse modulus ratios for $s_{T}=3$

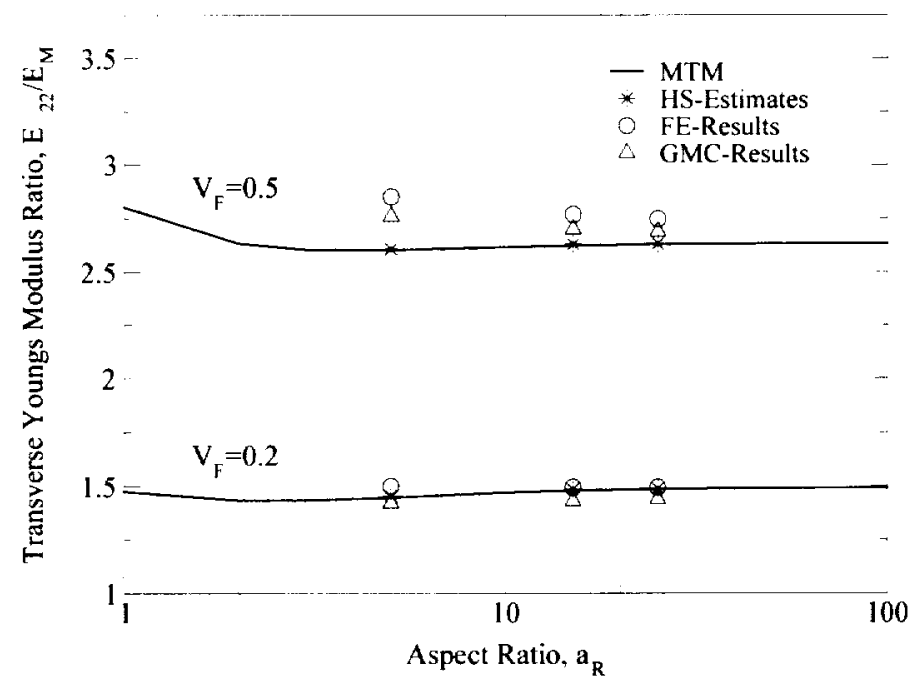

Figure 8: Comparison of transverse modulus ratios for $s_{r}=30$ 


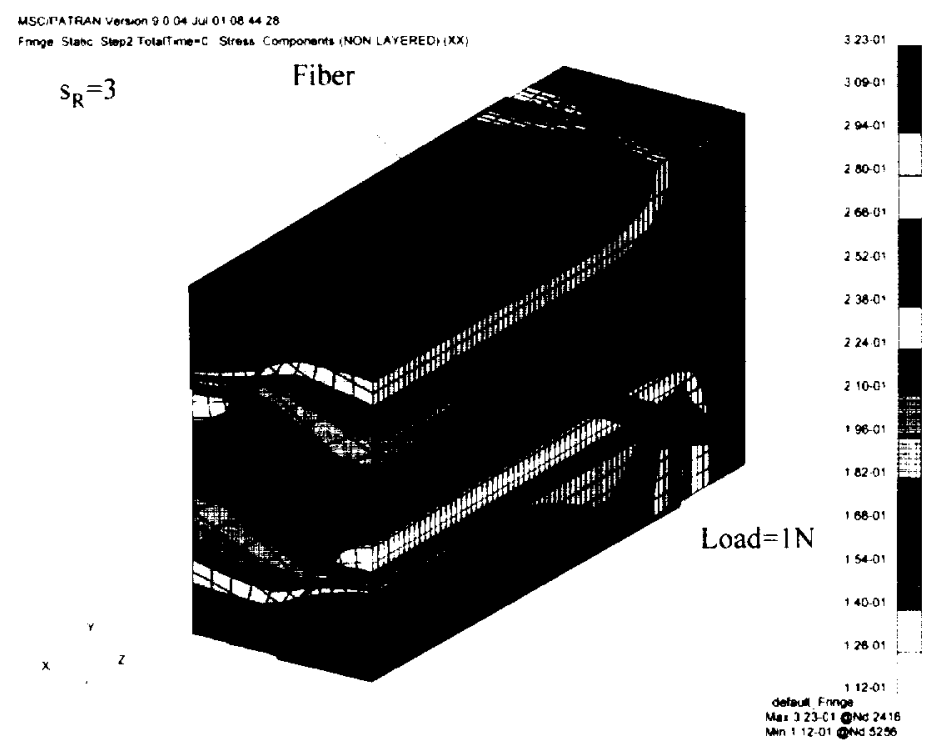

Figure 9: Example of transverse stresses obtained from FE-calculation for $s_{r}=3\left(V_{F}=0.2, a_{R}=5.0\right)$

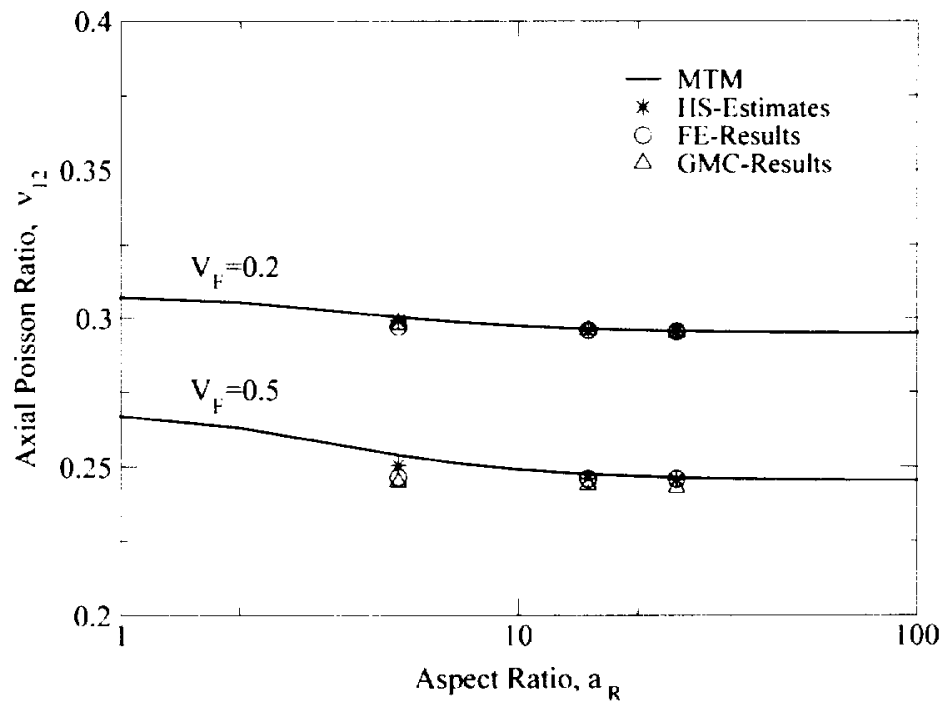

Figure 10: Comparison of axial Poisson ratios for $s_{r}=3$ 


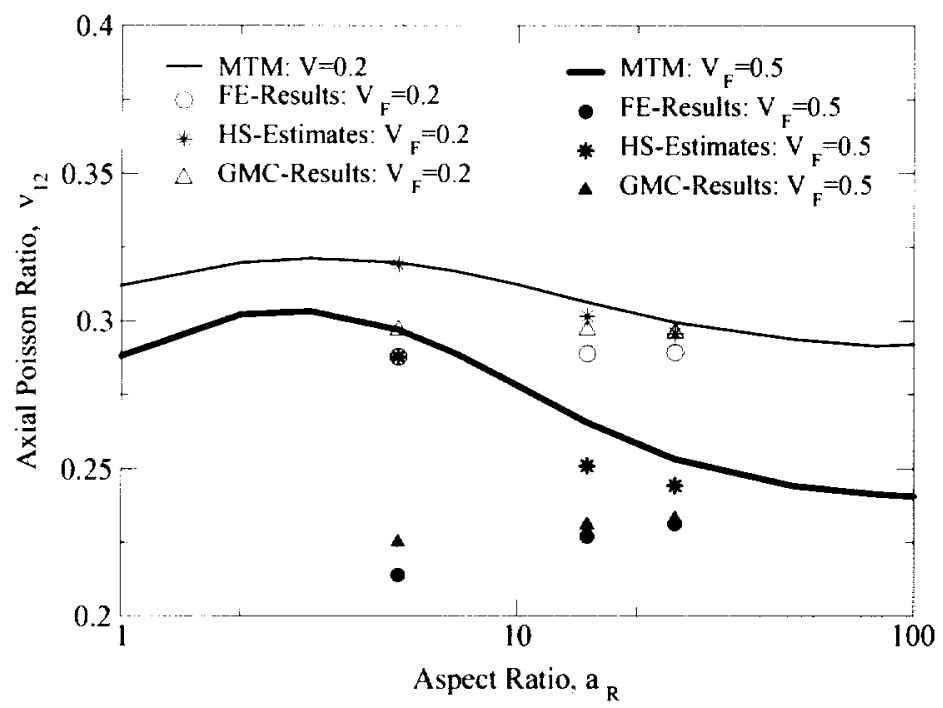

Figure 11: Comparison of axial Poisson ratios for $s_{r}=30$

Similarly, results for the in-plane Poisson's ratio $\nu_{23}$ appear in Figs. 12 and 13. Once again, in general the GMC results are slightly above those produced with FEA. The Hashin-Shtrikman estimates being closer to the FE-results than the GMC results. The discrepancy between methods being more evident with higher fiber volume fraction than higher stiffness ratios. Even so, the maximum error between the GMC predictions and those of FEA is at most $4.8 \%$.

\subsection{Elastoplastic Material Behavior}

In this section the elastoplastic stiffness behavior of aligned short-fiber composites will be analyzed using primarily the GMC and the finite element method. A comparison of the resulting predictions should confirm whether or not the GMC is capable of accurately simulating the elastoplastic stiffness behavior of DRX materials. Additionally, results obtained from a semi-analytical elastoplastic shear lag model (see Appendix B) are included as well to help illuminate any differences between the methods. The investigated model parameters are similar to those of the elastic investigation, with the exception that only two fiber aspect ratios $\left(a_{r}=5\right.$ and 25) are examined. Furthermore we restrict ourselves to the case of longitudinal ( $\mathrm{x}_{1}-$ direction) loading so as to allow the inclusion of shear lag results.

Figure 14 shows the effective longitudinal stress-strain curve produced by the GMC (dotted and dashed line), FE unit cell (solid line), and shear lag (symbols) models for the special case of $V_{f}=0.2, a_{r}=5, s_{r}=3$. Clearly, a large difference in the longitudinal stiffness response exists, depending upon the analysis method employed, with the GMC model predictions being significantly softer. The question before us is whether or not the lack of shear coupling inherent in the GMC method is the primary cause for the softer elastoplastic behavior. To address this question, we introduced a semi-analytical shear-lag-model (and shear-free model) as described in Appendix B. A comparison of the shear-lag (open circles) and shear-free (open square symbols) models with those of FE and GMC are also shown in Fig. 14.

Evidently, the 8-subcell ( 1 fiber subcell surrounded by 7 matrix subcells) GMC results and the shear-free model results agree extremely well; whereas, those determined using the elastoplastic shear-lag model agree with those coming from the FE unit cell model. Consequently, it may be concluded that:

1. The difference between the GMC- and FE-simulations is directly related to the lack of normal-shear coupling inherent in the GMC formulation. 


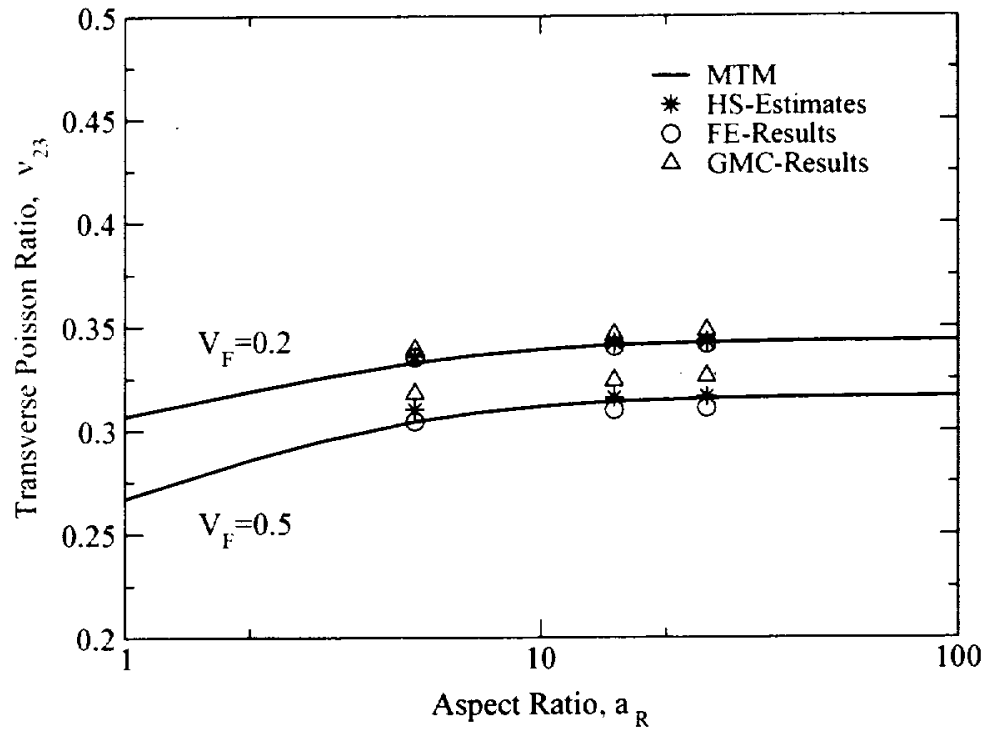

Figure 12: Comparison of transverse Poisson ratias for $s_{r}=3$

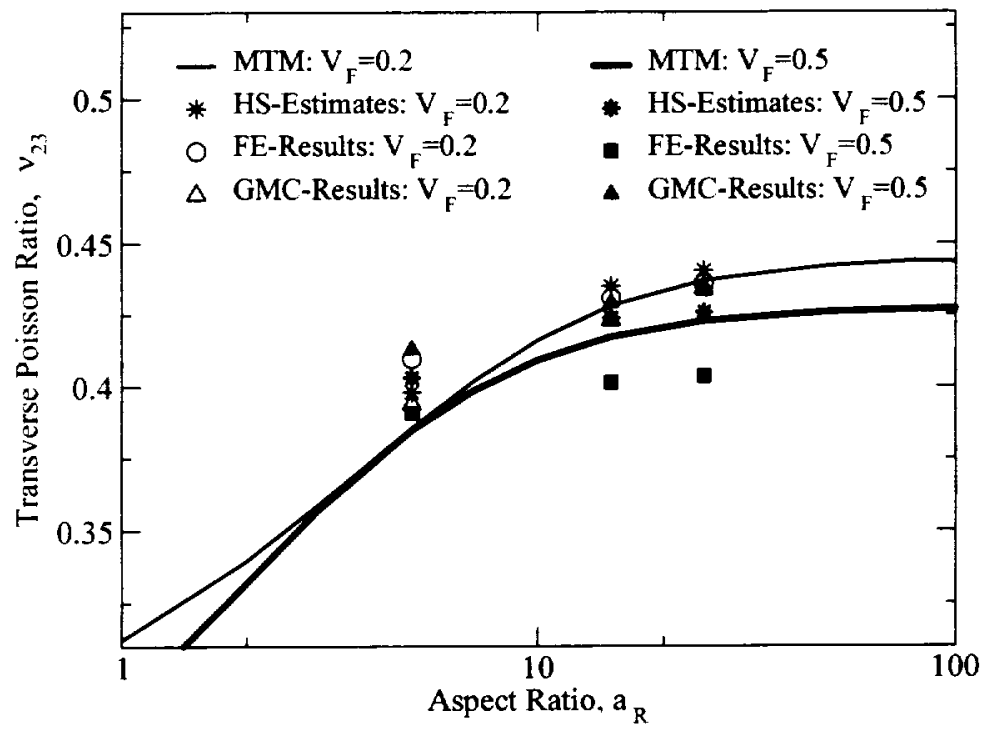

Figure 13: Comparison of transverse Poisson ratios for $s_{r}=30$ 


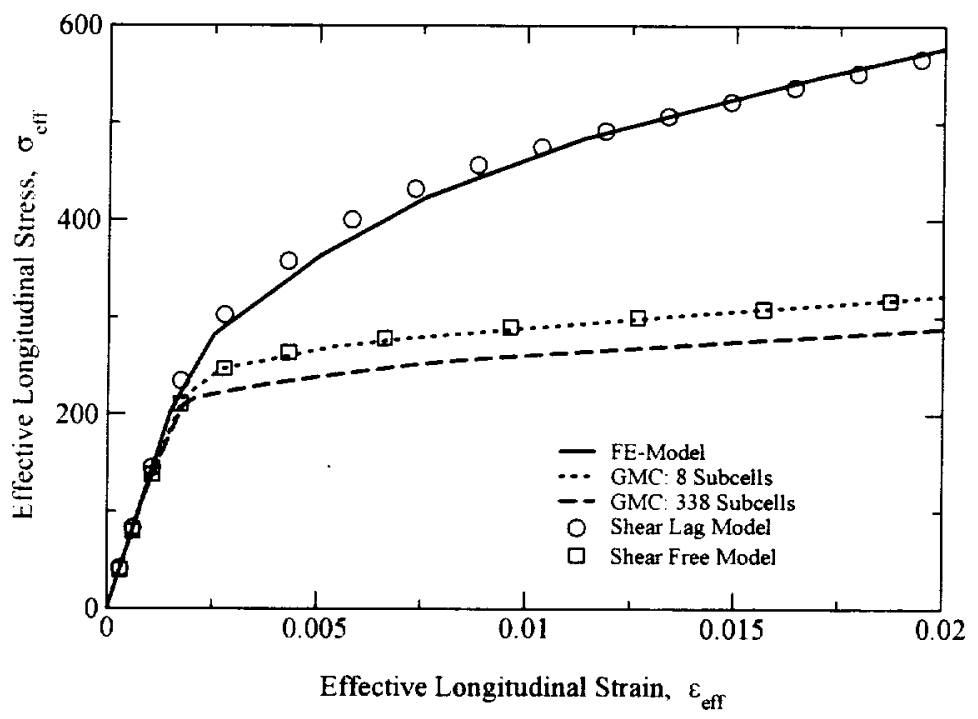

Figure 14: Comparison of FE-, GMC-, Shear-lag-, Shear-free-model results $\left(s_{r}=3, V_{f}=0.2, a_{r}=5\right)$

2. The standard (without any modifications) GMC-model is not well suited for predicting the elastoplastic response behavior of DRX materials, as the GMC-model predicts an overly soft plastic response.

3. The semi-analytical elastoplastic shear-free and shear-lag models introduced in Appendix B appear fully capable of predicting both the GMC- and FEA-results. The primary disadvantage of this shearlag model being its "uniaxial" nature, that is, it can only provide the effective modulus (response) in a single direction.

In addition to the pseudo-square ${ }^{3}$ fiber (8-suboell) GMC representation (dotted line) in Fig. 14, the typical (338 subcell) circular fiber unit cell (dashed line) is shown in Fig. 14. Clearly, a discretization effect is observed and is a result of the piecewise plastification of the matrix subcells between the two fiber ends in the case of the finer GMC-model. This effect results in a softer response within the initial plastic range (i.e., from $0.2-0.5 \%$ strain range). After plastification of all matrix subcells the effective hardening slope of both GMC unit cells are essentially the same.

To verify the above conclusions, simulations with other aspect ratios, volume fractions and stiffiness ratios were conducted with the results being displayed in Figs. $15-18$. The semi-analytical shear-free model is not shown in these figures as its results always agreed with the GMC (pseudo square) simulations. The fitting parameter $\psi$ of the semi-analytical shear-lag-model is found to be only a function of the fiber volume fraction i.e. $\psi=\psi\left(V_{f}\right)$ and takes a value of approximately 2 for $V_{f}=0.2$ and approximately 2.5 for $V_{f}=0.5$. The dashed lines in all figures represent the effective stress-strain response of the standard circular GMC-model. All figures confirm the above conclusions.

Some characteristic features of the effective finite element stress-strain curves in Figs. 15 - 18 are evident; for example, increasing either the aspect ratio or volume fraction significantly hardens the composite response, particularly for high stiffiness ratio materials. The elastoplastic stiffness response may be partitioned into three ranges. Figure 19 shows these ranges as a function of effective longitudinal strain.

Range 1, delineates the domain where linear elastic behavior is manifested This is followed by range 2 in which a large variation in the hardening slope takes place and range 3 in which approximately a constant

\footnotetext{
${ }^{3}$ In GMIC the geometry of the subcell representing the fiber is square: however in actuality since all fields are taken at the centroid of the subcell only the ratio of subcell to total length is important. no corners are felt. Thus the square fiber is really. a pseudo-square.
} 


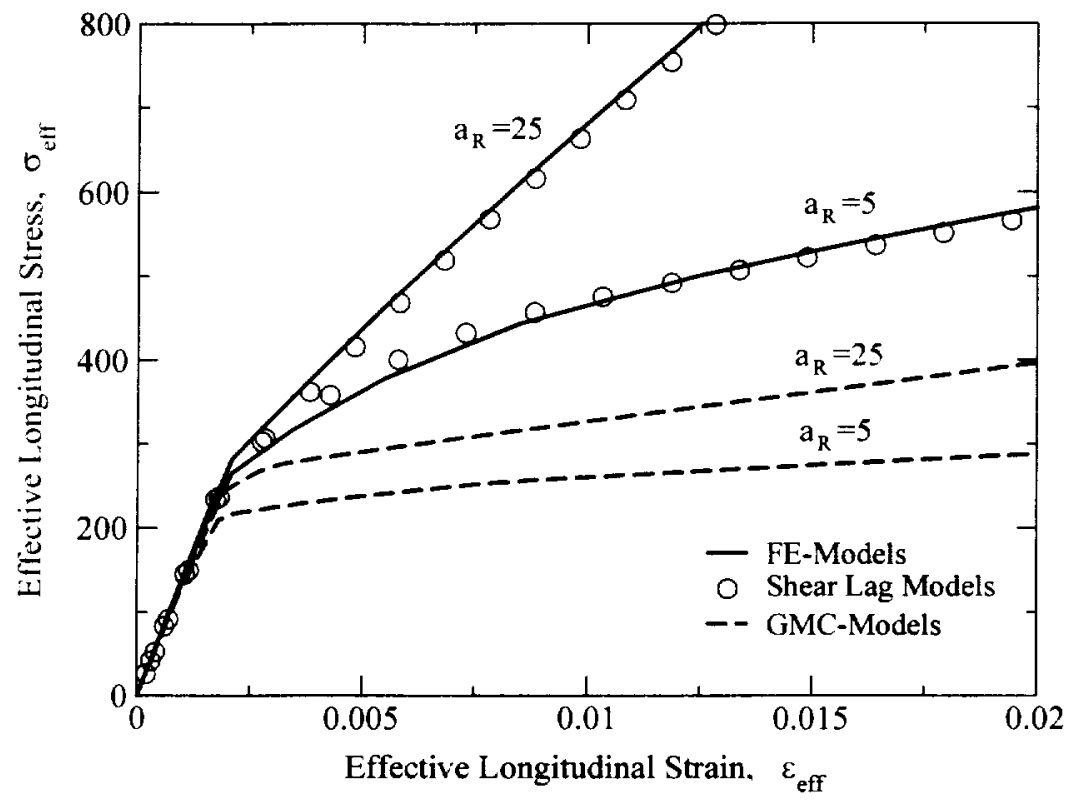

Figure 15: Comparison of FE-. GMC- and Shear-lag-model results for $s_{r}=3$ and $V_{f}=0.2$

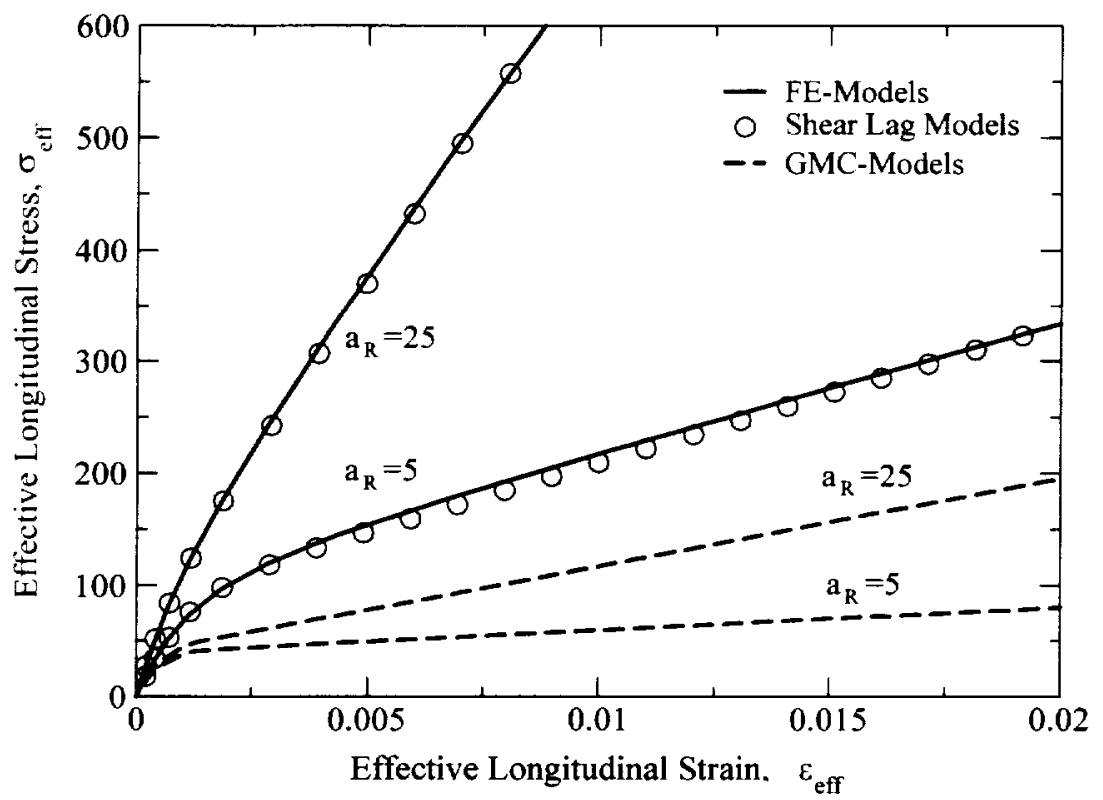

Figure 16: Comparison of FE-, GMC- and Shear-lag-model results $s_{r}=3$ and $V_{f}=0.5$ 


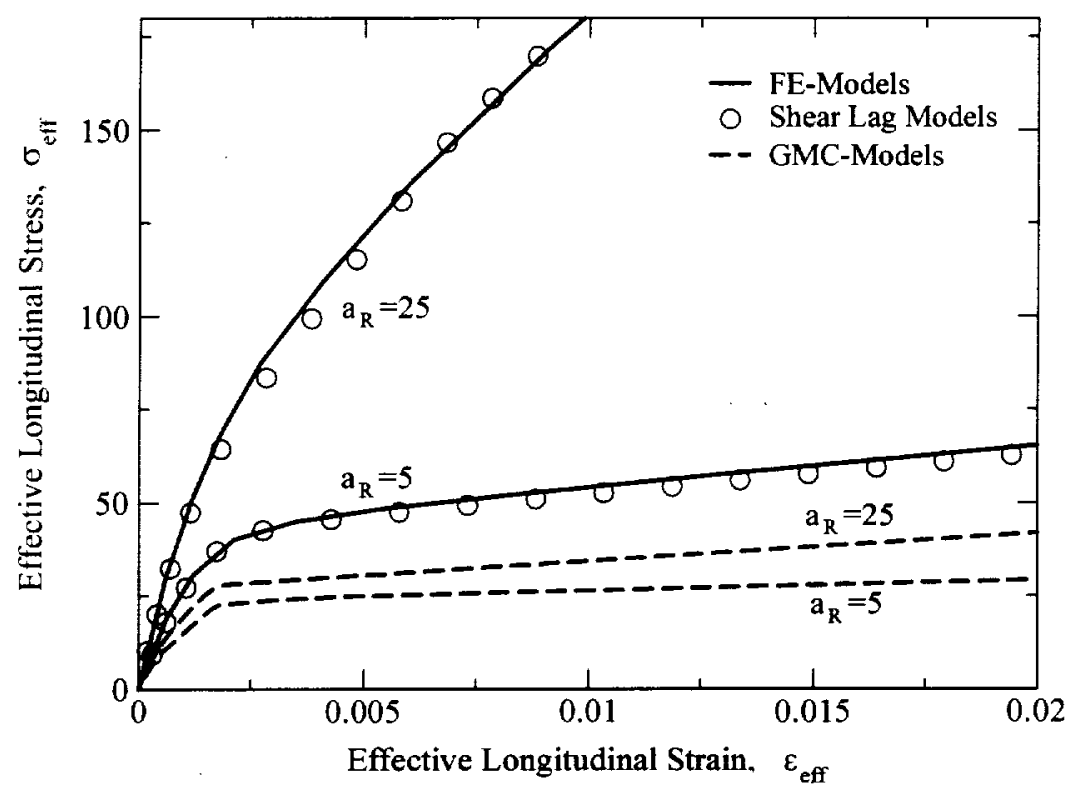

Figure 17: Comparison of FE-, GMC- and Shear-lag-model results $s_{r}=30$ and $V_{f}=0.2$

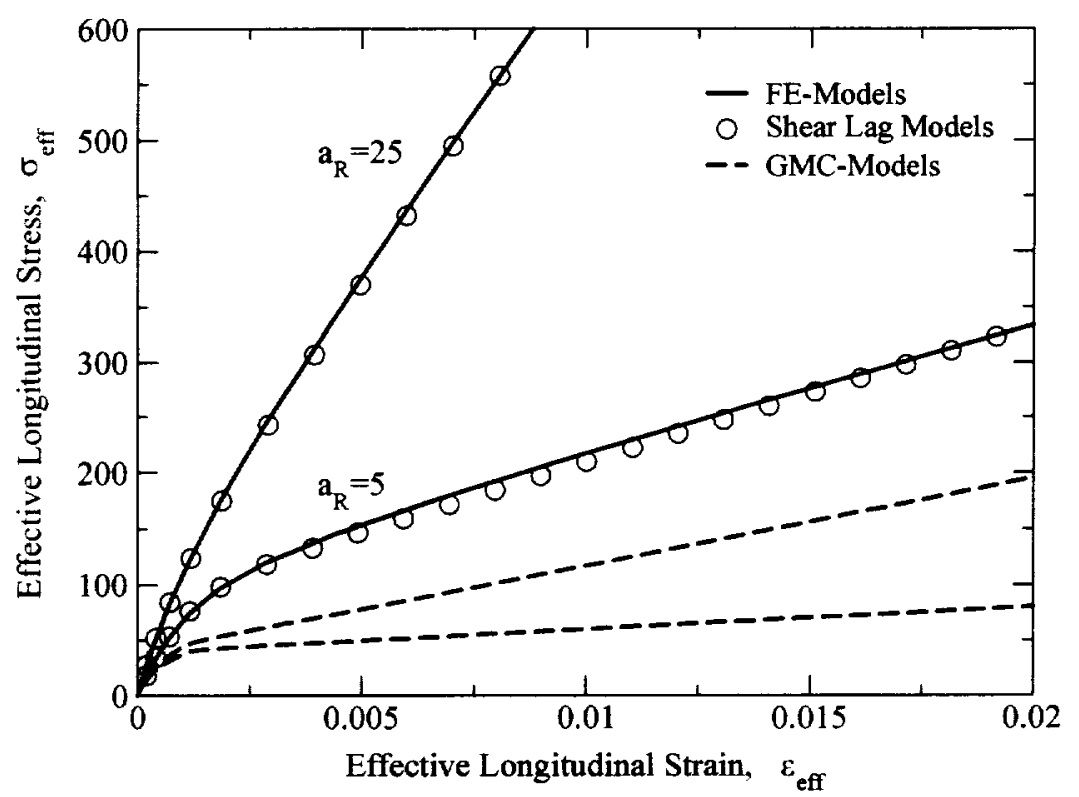

Figure 18: Comparison of FE-, GMC- and Shear-lag-model results $s_{r}=30$ and $V_{f}=0.5$ 


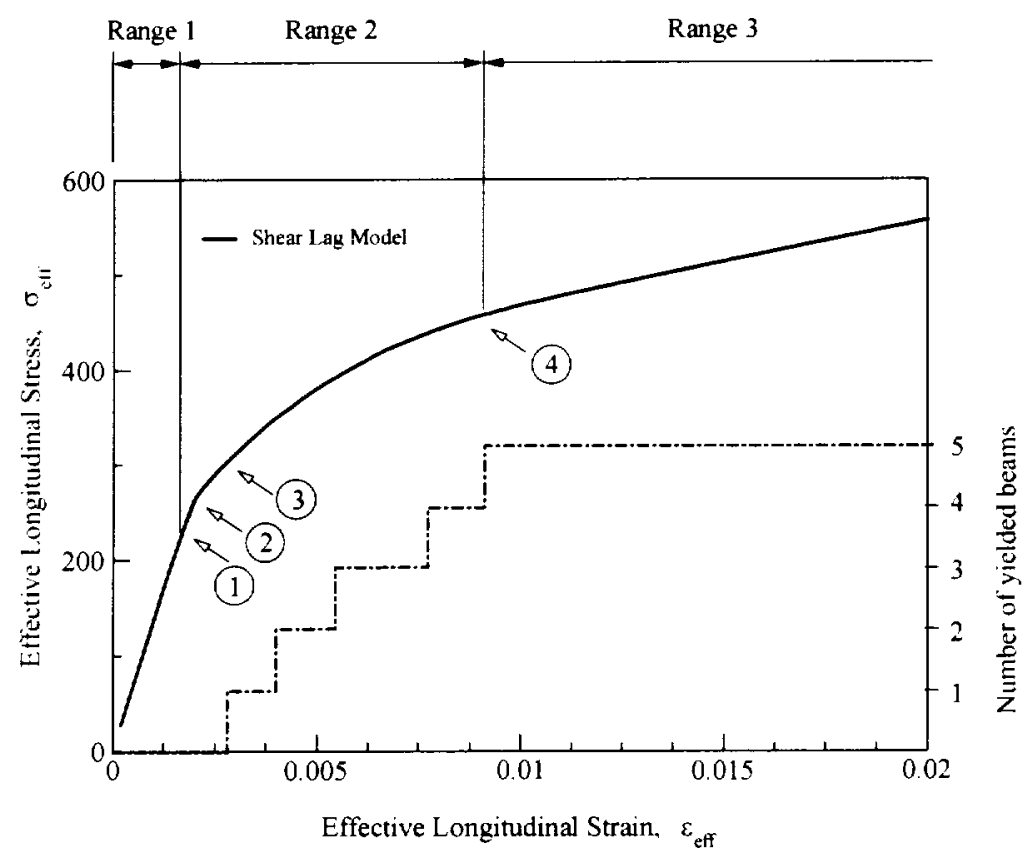

Figure 19: Semi-analytical shear-lag model, characteristic ranges and number of yielded truss elements $\left(s_{r}=3, V_{f}=0.2, a_{r}=5\right)$

effective hardening slope is observed. More detailed investigations into the flow behavior show that at the beginning of range 2 the matrix material between the two fiber ends begins to yield first (see Fig. 19, point 1). After which the whole matrix yields (point 2). During the longitudinal loading step the matrix fron the side-to-sicle region tries to move to the end-to-end region. This material flow leads to a considerable shear loading of the side-to-sicle matrix interphase (between fiber and matrix). In the plastic range large shear deformations of the interphase start at the fiber ends (point 3), then grow over the fiber and prevent any additional loading of the fiber. In the case of the semi-analytical shear-lag-model this means that one by one each non-linear truss element will start to yield. All trusses are plastic at point 4 (see Fig. 19), which is the beginning of range 3. Note, the stair-stepped dashed line in Fig. 19 indicates the piecewise yielding of, altogether, five truss elements.

Based on these results, the question remaining is whether the GMIC can be appropriately modified in order to predict accurately the elastoplastic behavior of DRX materials. Considering why the GMC fails. it becomes clear that if the matrix between the two fiber ends yields, the fiber is no longer capable of carrying any additional load beyond the matrix since both the matrix and fiber have the same stress state. This continuity of specific stress components along a given row or column of subcells is a direct result of the inherent lack of normal and shear coupling with GMC. Given this fact, a simple modification to GMIC would be the intraduction of an artificial matrix material for all subcells within the region between the two fiber ends. This new artificial matrix material must be chosen so that the resulting effective stress-strain curve produced corresponds with the reference data (be it experimental or analytical). Of course this strateg possesses the drawback that the obtained micro-mechanical GMC stress state is no longer based on any physical meaning: i.e., fracture, damage or fatigue analyses could no longer be performed confidently when using the modified GMIC-results, as the microfield quantities would be even more questionable than before.

As a first attempt, the artificial matrix material was assumed to behave as purely elastic. with the associated Young's modulus of the artificial matrix material being that of the matrix itself. Clearly; such an assumption results in the overestimation (at least well within the plastic range) of the elastoplastic response curve as illustrated in Fig. 20. This response prediction is non-conservative. In contrast. it is shown that the 


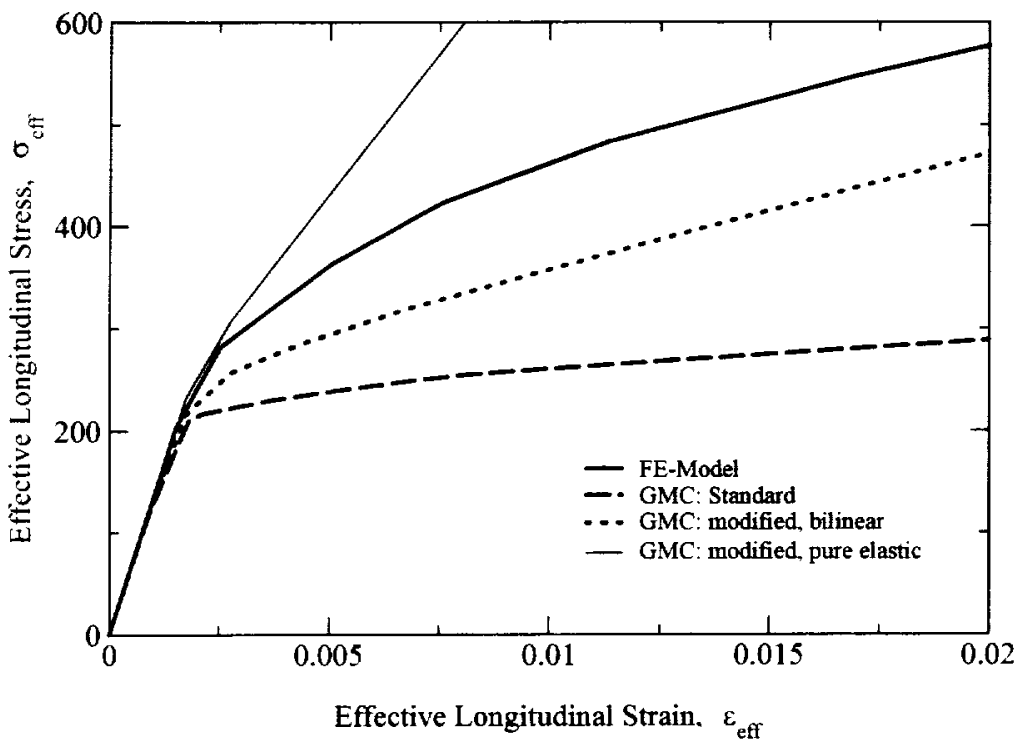

Figure 20: Comparison of FE-, GMC-, and GMC-modified simulations $\left(s_{r}=3, V_{f}=0.2, a_{r}=5\right)$.

standard GMC-model provides a lower bound (conservative response prediction) for the effective elastoplastic behavior, thus suggesting that an artificial bilinear elastoplastic material might provide a more accurate simulation. For such a material definition, four material parameters would be recuired as input; they are 1) Poisson's ratio, 2) Young's modulus, 3) yield stress, and 4) the plastic hardening slope. With the yield stress and hardening slope being the best candidates for modification from that of the matrix material, since the elastic range is already accurately predicted, the parameters $(E, \nu)$ can be taken to be that of the matrix material. Here we restrict ourselves to the modification of the hardening slope alone (keeping the yield the same as the of the matrix) and thereby attempt to obtain the unknown artificial hardening slope $E_{H}^{*}$ from the consideration of a simple one dimensional spring model (with each spring corresponding to a given subcell) which is shown in Fig. 21.

The following discussion is limited to range 3 in Fig. 19 (i.e., the range where the effective hardening slope is approximately constant). Assume an incremental load, $\Delta F$, is applied to the spring assemblage in Fig. 21. This results in an incremental displacement, $\Delta u$, that depends on both the geometry and material properties of the spring assemblage, that is,

$$
\Delta F=\left(\frac{1}{\frac{l_{f}}{\bar{E}_{f} A_{f}}+\frac{l_{f}}{E_{H}^{*} A_{f}}}+\frac{1}{\overline{E_{H}\left(A-A_{f}\right)}}\right) \Delta u,
$$

where $A$ is the total crass sectional area of the considered unit cell and $A_{f}$ the fiber cross sectional area. $E_{H}$ and $E_{H}^{*}$ are the hardening slope and artificial hardening slope, respectively. Alternatively; the global longitudinal stiffiness behavior can be described by

$$
\Delta F=\frac{E_{H}^{e f f} A}{l} \Delta u
$$

where $E_{H}^{e f f}$ is the effective longitudinal hardening slope. The combination of Eqs. (2) and (3), and the introduction of the following abbreviations,

$$
s_{r, H}^{f}=\frac{E_{f}}{E_{H}}, \quad s_{r, H}^{e f f}=\frac{E_{H}^{e f f}}{E_{H}}, \quad l_{r}=\frac{l_{f}}{l}, \quad V_{f}=\frac{A_{f} l_{f}}{A l}
$$




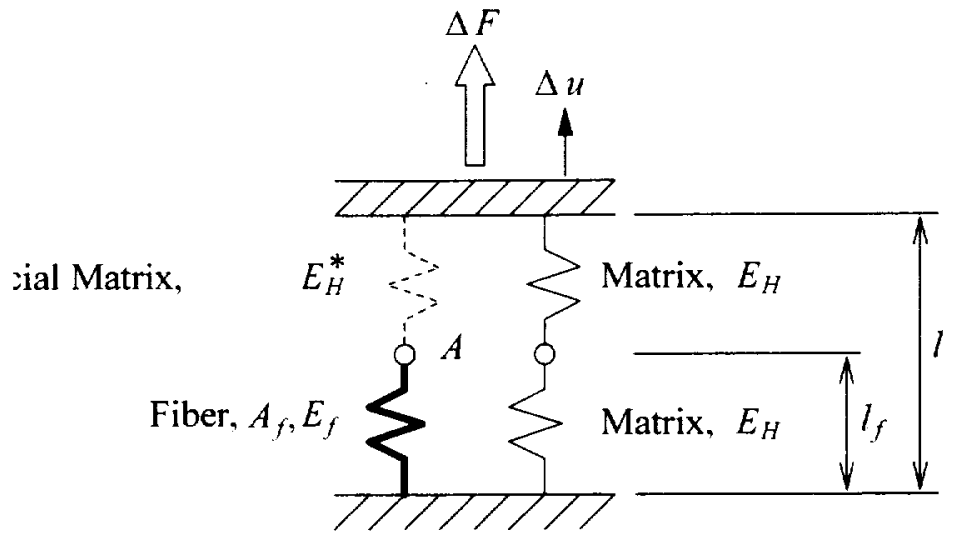

Figure 21: Spring assemblage model

lead to the final equation for the artificial hardening slope:

$$
\frac{E_{H}^{*}}{E_{H}}=\frac{s_{r . H}^{f}\left(1-l_{r}\right)\left(s_{r . H}^{e f f}+\frac{v_{f}}{l_{r}}-1\right)}{V_{f}\left(\frac{s_{r, I}^{f}}{l_{r}}-1\right)+l_{r}\left(1-s_{r . H}^{e f f}\right)} .
$$

Note, the only unknown in this equation is the effective longitudinal hardening slope $E_{H}^{e f f}$ which for example may be determined from the semi-analytical shear-lag model (see Appendix B) or obtained from some other reference data.

A comparison of the FE-. GMC-, GMC-bilinear-modified-model response histories are shown in Figs. 2225. Evidently, as one might expect. the GMIC-model, modified by using an artificial bilinear matrix material definition in the intermediate subcell, results in a more accurate longitudinal simulation than the previous standard GMC-model (see Fig. 14 and that modified using an artificial elastic matrix material definition). With this approach, reasonably accurate results can be produced when range 2 is small, ty pically for high fiber aspect ratios and low stiffness ratios. Alternatively; for situations involving high stiffness ratio materials. low volume fractions and high aspect ratias (e.g., $a_{r}=25$, see Fig. 24) simulations using the modified GMC approach give the largest error. This is due to the fact that these conditions induoe the smallest area (highly loaded) suboell between fibers and thus maximizes the flow between inclusions; thereby producing the most compliant overall response. Clearly, only a small difference exists between FE and GMC results in the extensive plastic range. This is due to the fitting procedure utilized for the simple spring model. Note that, again all response histories are conservative when compared with FEA simulations.

More detailed studies were not performed at this time using a more complex artificial matrix material definition, since the actual usefulness of such an ad-hoc approach is debatable due to the lack of any real physical meaning. Furthermore, a new higher order GNC theory (appropriately termed - high fidelity GMC), which provicles the necessary normal and shear coupling, has recently been developed by. [A boudi et. al.(2001)]. This new formulation, although more computationally demanding than the standard or modified GMC (but significantly faster than the FEA approach) should provide the required balance between speed and accuracy when at tempting to simulate DRX material behavior. Consequently; further analysis using the GMC will be reserved until later when the theoretical extension of the present elastic continuously reinforced high fidelity GMC version (see [Aboudi et. al.(2001)]) to that of discontinuous and inelastic material behavior, is acoomplished. 


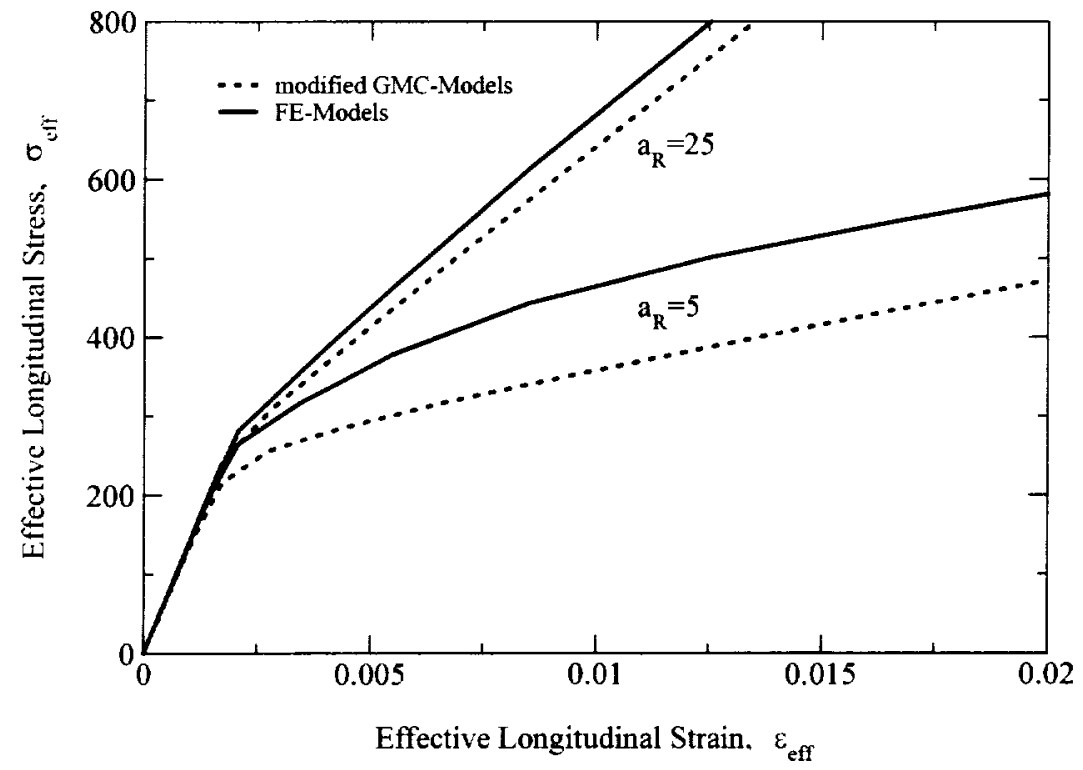

Figure 22: Comparison of FE- and GMC-modified model $\left(s_{r}=3, V_{f}=0.2\right)$

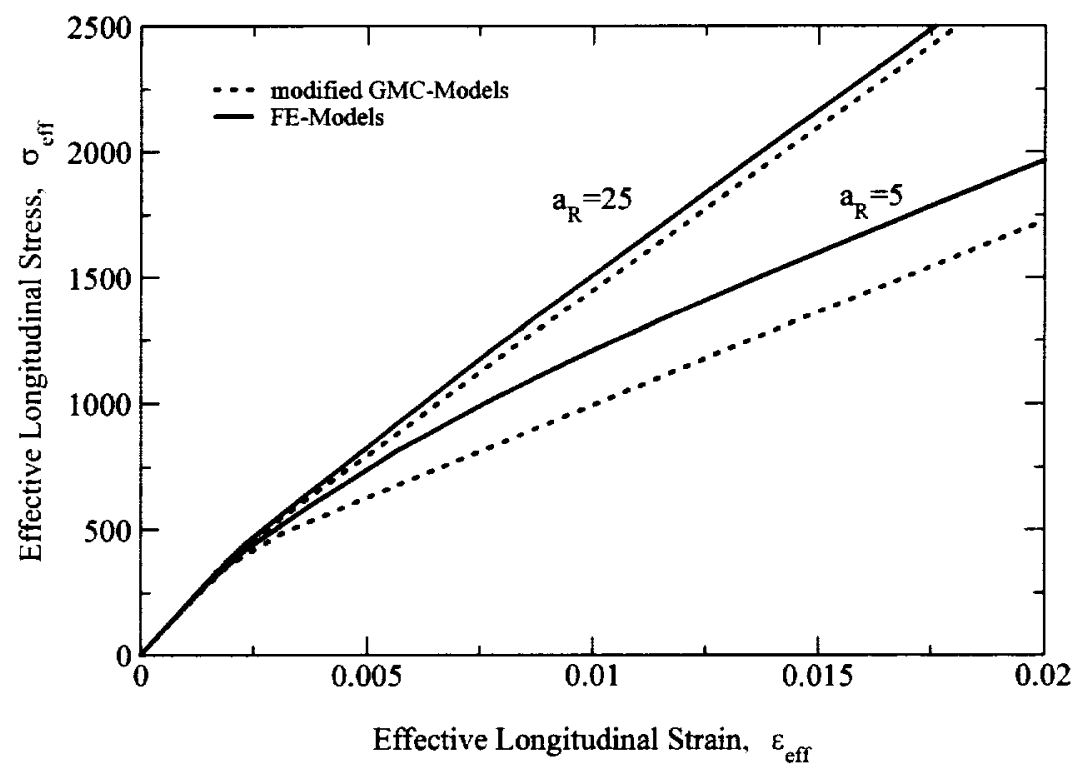

Figure 23: Comparison of FE- and GMC-modified model simulations $\left(s_{r}=3, V_{f}=0.5\right)$ 


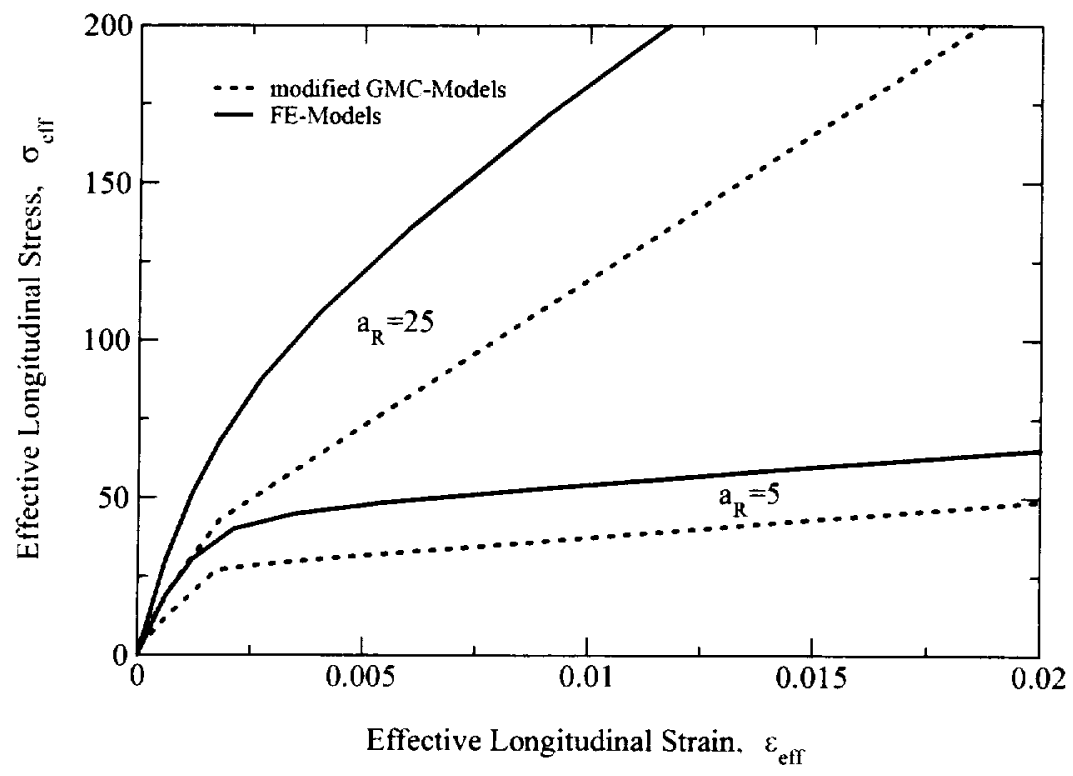

Figure 24: Comparison of FE- and GMIC-modified model simulations $\left(s_{r}=30, V_{f}=0.2\right)$

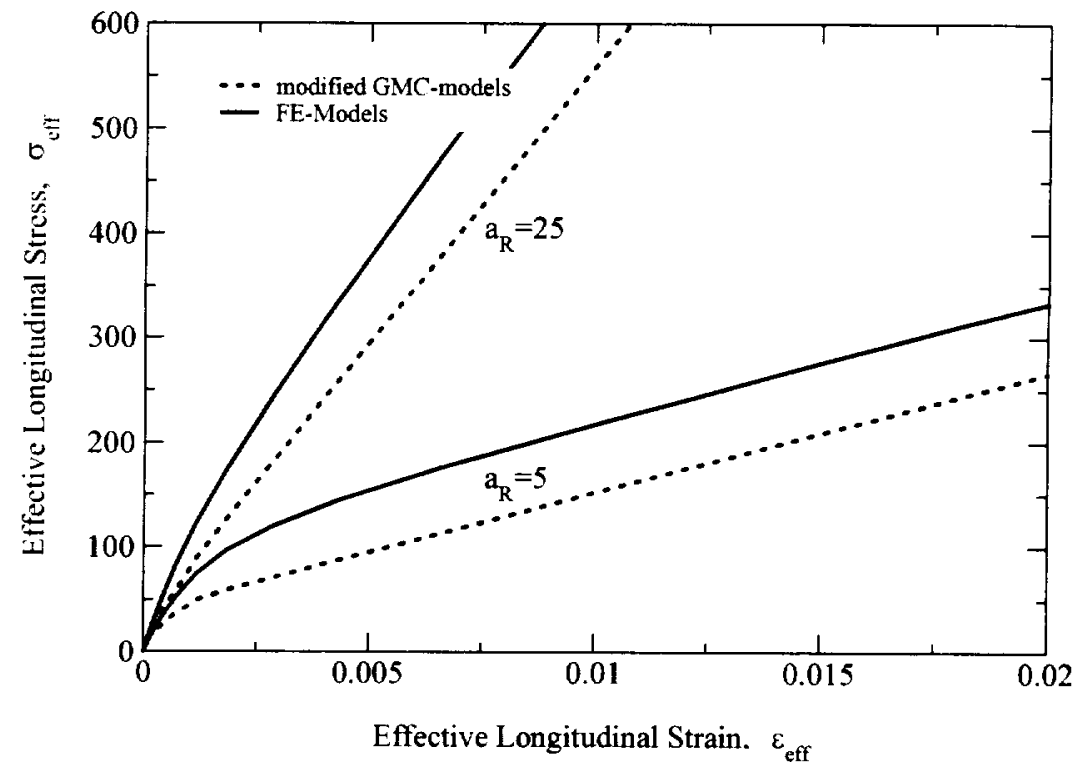

Figure 25: Comparison of FE- and GMIC-modified model sinulations $\left(s_{r}=30, V_{f}=0.5\right)$ 


\section{Conclusions}

This paper has focused on evaluating the predictive ability of the generalized method of cells, in the context of discontinuously reinforced compasite (DRX) material behavior; specifically aligned short fiber compasites. GMIC simulations were compared with: i) simulations using other micromechanical based mean field models and finite element (FE) unit cell models found in the literature for the elastic range, as well as ii) finite element unit cell model and a new semi-analytical elastoplastic shear lag model in the inelastic range. Results indicated that the GMC is fully capable of accurately analyzing the elastic behavior of DRX materials. However, in the case of inelastic behavior, the standard GMIC approach, due to the inherent lack of normal-shear coupling, was shown to be unsuitable for predicting the longitudinal elastoplastic behavior of aligned short fiber reinforoed compasites. However, a simple modification of the GMC-model, through the introduction of an "artificial" bilinear matrix material definition within the subcell(s) residing between inclusion (fiber) ends was shown to improve the overly conservative GMC predictions within the elastoplastic regime significantly. This ad-hoc modification to the GMC unit cell definition would allow reasonably: accurate multiaxial simulations to be made. The case when both the stiffness and fiber aspect ratios are high being the least accurate. Finally; it was shown herein that an elastoplastic shear lag model can provide identical uniaxial results to that of the FE unit cell calculations. For truly consistent and accurate results it is recommended that a high fidelity micromechanics approach be used when examining inelastic discontinuously reinforced compasite behavior.

\section{References}

ABAQUS (1998). Standand User's Manual, Version 5.8. Hibbit, Karlsson \& Sorensen, Inc., Pawtucket, RI, USA.

Abel, C. A., Weissenbek, E. and Rammerstorfer, F. G. (1993). Influence of damage on the mechanical behavior of short fiber reinforced MMCs with strong and weak interfaces. ZAMM 73, pp. T423-T426.

Aboudi, J. (1991) Mechanics of Compasite Materials, A Unified Micromechanical Approach, Studies in Applied Mechanics, 29, Elsevier.

Aboudi, J. (1995). Micromechanical analysis of thermo-inelastic multiphase short-fiber compasites. Comp. Eng. 5, pp. 839-850.

Aboudi, J. (1996). Micromechanical analysis of compasites by the method of cells - Update. Appl. Mech. Rev. 49, No. 10, pp. S\$3-S91.

Aboudi, J., Pindera, M.J. and Arnold, S.M. (2001) Thermoelastic Higher-Order Theory for Periodic Multiphase Materials. Jnl. Of Applied Mech., to appear 2001

Arnold, S. M.; Pindera, M. J.; and Wilt, T.E. (1996) Influence of Fiber Architecture On The Inelastic Response of Metal Matrix Composites. Int. Jnl. of Plasticity, 12, No. 4, pp. 507-545.

Amold, S. M., Bednarcyk, B. A., Wilt, T. E. and Trowbridge, D. (1999). Micnomechanical analysis code with generalized method of cells (MAC/GMC), user guide: version 3.0. NASA/TM-1999-209070, Cleveland, USA.

Ashton, J. E., Halpin, J. C. and Petit, P. H. (1969). Primer on composite materials: Analysis. Technomic Publishing Co, Stamford, Conn. pp. 77-81. 
Bao, G., Hutchinson, J. W. and McMeeking, R. M. (1991). Particle reinforcement of ductile matrices against plastic flow and creep. Acta Metall. Mater. 39, pp. 1871-1882.

Bednarcyk, B. A., and Arnold, S. M. (2000a) A New Local Failure Model With Application to the Longitudinal Tensile Behavior of Continuously Reinforced Titanium Composites NASA/TM 2000-210027.

Bednareyk, B. A., and Arnold. S. M. (2000b) A New Local Debonding Mlodel With Application to the Transverse Tensile and Creep Behavior of Continuously Reinforoed Titanium Composites NASA/TMI 2000210029

Benveniste, Y. (1987). New approach to the application of Mori-Tanaka's theory in composite materials. Mech. Mat. 6. pp. 147-157.

Brown, L. M. and Clarke, D. R. (1975). Work hardening due to internal stresses in compasite materials. Acta Metall. 23, pp. 821-830.

Christman, T., Needleman, A. and Suresh, S. (1989). An experimental and numerical study of deformation in metal-ceramic composites. Acta Metall. Mater. 37, pp. 3029-3050.

Cox, H. L. (1952). The elasticity and strength of paper and other fibrous materials. Brit. J. Appl. Phys $\mathbf{3}$, pp. $72-79$.

Clyne, T.W. and Withers, P.J. (1993) An Introduction To Metal Matrix Composites, Cambridge University Press.

Dow, N. F. (1963). No. R63-SD61 . Technical report, General Electric Report.

Dragone, T. L. and Nix, W. D. (1960). Geometric factors affecting the internal stress distribution and high temperature creep rate of disoontinuous fiber reinforced metals. Acta Metall. Mater. 38, pp. 1941-1953.

Eshelby; D. (1957). The determination of the elastic field of an ellipsoidal inclusion and related problems. Proc. Royal Soc. (London) A 241, pp. 376-396.

Goldberg, R.K and Amold. S. M. (2000) A Study of Influencing Factors on the Tensile Response of a Titanium Matrix Compasite with Weak Interfacial BondingNASA/TM 2000-209798.

Hill, R. (1965). A self-consistent mechanics of composite materials. J. Mech. Phys. Solids 13, pp. 213-222.

Hu, G. K. and Weng, G. J. (2000). The connections between the double-inclusion model and the Ponte Castaneda-Willis, Mori-Tanaka, and Kuster-Toksoz models. Mech. Mat. 32, pp. 495-503.

Iyer, S.K., Lissenden, C.J., and Amold, S.M. (2000) Local and overall flow in composites predicted by. micromechanics Composites: Part B, 31, pp. 327-3+3.

Kelly, A. and Zweben, C. (Eds) (2000) Comprehensive Compasite Materials, Volume 3: Netal Matrix Composites ISBN: 0-080437214, Pergamon.

Levy, A. and Papazian, J. M. (1990). Tensile properties of short fiber-reinforced SiC/Al compasites - Part II: Finiteelement analysis. J. Appl. Mech. 21A. pp. $411-420$.

Lissenden, C.J., Arnold, S.M., and Iyer S.K. (2000) Flow/damage Surfaces for Fiber-Reinforced Metals having Different Periodic Micrastructures Int. J. Plasticity, 16, No. 9, pp. 10ł9-107t.

Lloyd, D.J. (1994) Particle Reinforoed Aluminium and Magnesium Matrix Composites, Int. Material Reviews, 39, No. 1, pp. 1-23

Miracle, D.B. and Maruyama, B. (2000) Metal Matrix Composites for Space Systems: Current Uses and Future Opportunities, Proc. National Space and missile Materials Sympasium, Ed, M. Stropki, Dayton, OH: Anteon Corp., pgss 25. 
Mori, T. and Tanaka, K. (1973). Average stress in the matrix and average elastic energy of materials with misfitting inclusions. Acta Metall. 21, pp. 571-574.

Paley; M. and Aboudi, J. (1992). Micromechanical analysis of composites by the generalized cells model. Mech. Mat. 14, pp. 127-139.

Pindera, M-J, and Bednarcyk, B.A., (1999) An Efficient Implementation of the Generalized Method of Cells for Unidinectional, Multi-Phased Composites With Complex Micrastructures Compasites Part B, 30, No. 1, pp. 87-105.

Pedersen, O. B. and Withers, P. J. (1992). Iterative estimates of internal stresses in short-fiber metal matrix composite. Phil. Mag. A65, pp. 1217-1233.

Ponte Castañeda, P. and Willis, J. R. (1995). The effect of spatial distribution on the effective behavior of composite materials and cracked media. J. Mech. Phys. Solids 43, pp. 1919-1951.

Povirk, G. L., Needleman, A. and Nutt, S. R. (1990). An analysis of residual stress formation in whiskerreinforced Al-SiC compasites. J. Mat. Sci. and Eng. A125, pp. 129-140.

Siegmund, T., Weissenbek, E., Fischer, F. D. and Rammerstorfer, F. G. (1992). Micromechanical consideration of topological aspects regarding thermocyclic behatior. in The Processing, Properties and Applications of Metallic and Ceramic Materials II, pp. 971-976.

Sinclair, I. and Gregson, P.J. (1997) Structural Performance of Discontinuous Metal Matrix Compasites, Mat. Sci. and Tech., 13, pp. 709-726.

Tucker, C. L. and Liang, E. (1999). Stiffness predictions for unidirectional short-fiber composites: Review and evaluation. Comp. Sci. and Tech. 59, pp. 655-671.

Tvergaard. V. (1990). Analysis of tensile properties for a whisker-reinforced metal-matrix composite. Acta Metall. Mater. 38, pp. 185-194.

Weissenbek, E., Bøhm, H. J. and Rammerstorfer, F. G. (1993). Microgeometrical effects on the elastoplastic behavior of particle reinforoed MMCs. In: Proc. of 9th Int. Conf. on Composite Materials (ICCM/9 '93), July 12-16, 1993, Madrid, Spain.

Weissenbek, E. and Rammerstorfer, F. G. (1993). Influence of the fiber arrangement on the mechanical and thermo-mechanical behavior of short fiber reinforced mmes. Acta Metall. Mater. 41, pp. 2811-2843.

Weng, G. J. (1990). The theoretical connection between Mori-Tanaka theory and Hashin-Shtrikman-Walpole bounds. Int. J. Eng. Sci. 28, pp. 1111-1120.

Willis J. R., (1977). Bounds and Self-Consistent Estimates for the Overall Properties of Anisotropic Compasites; J. Mech. Phys. Sol. 25, 185-202.

Yang, J., Pickard. S. M., Cady; C., Evans, A. G. and Mehrabian, R. (1991). The stress-strain behavior of aluminum matrix composites with discontinuous reinforcements. Acta Metall. Mater. 39, pp. 1863-1869. 


\section{Appendix A: Generalized Method of Cells Formulation}

GNIC can predict the elastic and inelastic thermomechanical response of both continuous and discontinuous multiphased compasite materials with an arbitrary internal micrastructure and reinforcement shape. It is a continuum-based micromechanics model that provides closed-form expressions for the macroscopic compasite response in terms of the properties, size, shape, distribution, and response of the individual constituents or phases that make up the material ${ }^{t}$. These constituent materials can be represented using any. elastic and/or inelastic deformation and life (e.g., continuum damage mechanics fatigue model) model. The periodic nature of compasites typically allow's identification of a repeating unit cell that can be used as a building block to construct the entire compasite. The properties of this unit cell are thus representative of the properties of the entire assemblage once the subcell dinensions and appropriate subcell materials are selected. Thus, unidirectional long-fiber composites, short-fiber composites, porous materials and laminated materials can all be modeled as special cases.

\subsection{Model Description}

Consider a compasite material with a periodic structure whase repeating volume element consists of $N_{\mathrm{a}} \times N_{3} \times N$, rectangular parallelepiped subcells. The volume of each one of the subcells is $d_{\mathrm{a}} h_{3} l_{2}$, where a, 3 and, are running indices: $a=1 \ldots \ldots N_{a}: 3=1 \ldots N_{3} ; \uparrow=1 \ldots, N_{1}$, in the $x_{1}, x_{2}$ and $x_{3}$ directions, respectively: The total volume of the repeating volume element is $d h l$ where $d=\sum_{a=1}^{N_{a}} d_{a}$. $h=\sum_{3=1}^{N_{3}} h_{3}, C=\sum_{c=1}^{N_{1}} c_{\gamma}$. In Fig. 26, an example is shown for a repeating volume element with $N_{a}=2$. $N_{3}=3$ and $N_{3}=3$.

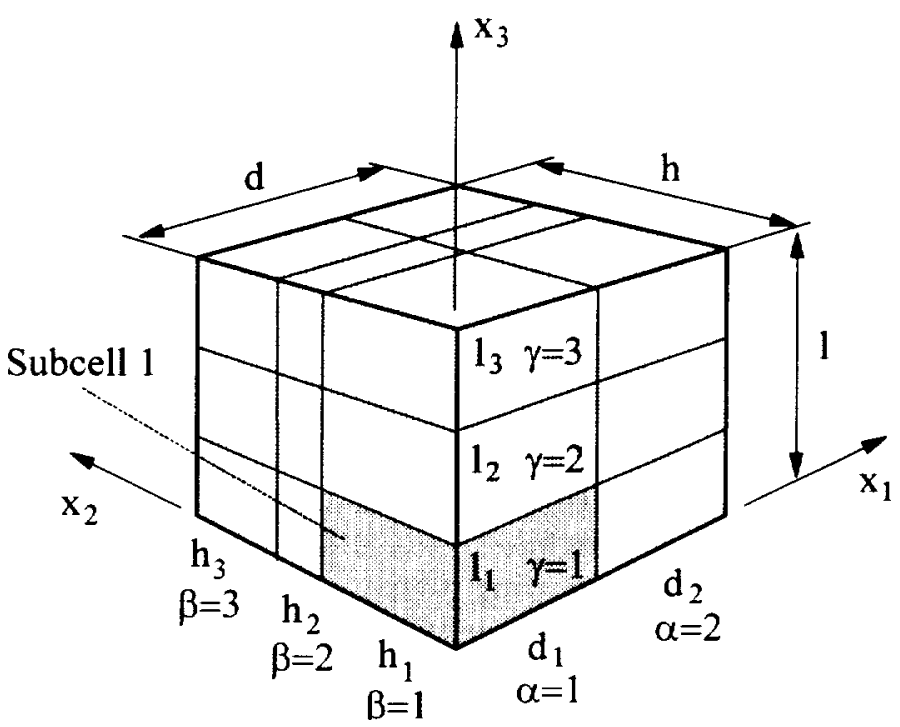

Figure 26: Triply periodic repeating unit œll, illustrating subcell dimension nomenclature.

By an approximate micromechanical analysis of the detailed interactions of the subcells of the repeating volume element, overall constitutive relations which govern the effective behavior of the multiphase elastoplastic composite, can be established. This analysis relies on the requirements that static equilibrium of the materials in the various subcells are ensured, and that continuity of the displacements and tractions between neighboring suboells within the repeating volume element, as well as between neighboring repeating volume elements is satisfied on an average basis.

\footnotetext{
${ }^{\dagger}$ Note. each of the subcells can be comprised. in general. by an elastic-viscoplastic temperature -dependent material.
} 
As the average behavior of the compasite is sought, it is sufficient to consider a first order theory in which the displacements $u_{i}^{\left.(a)_{1}\right)}$ in the subcells are expanded linearly in terms of the distances from the center of each subcell, i.e., in terms of the local coordinates $\bar{x}_{1}^{(a)}, \bar{x}_{2}^{(3)}$, and $\bar{x}_{3}^{(\gamma)}$. Thus, the first order expansion in the subcell $\left(a 3_{1}\right)$ is represented as,

$$
u_{i}^{(\mathbf{a}\})}=w_{i}^{\left.(\mathbf{a}\}^{\gamma}\right)}(\mathbf{x})+\bar{x}_{1}^{(\mathbf{a})} \phi_{i}^{(\mathbf{a} 3 \gamma)}+\bar{x}_{2}^{(3)} \chi_{i}^{(\mathbf{a} 3 \gamma)}+\bar{x}_{3}^{(\gamma)} \psi_{i}^{\left(\mathbf{a} 3_{\gamma}\right)} \quad i=1,2,3
$$

where $w_{i}^{\left(a_{3}\right)}(\mathbf{x})$ are the displacement components at the center of the subcell, and $\phi_{i}^{\left(a 3_{1}\right)}, \chi_{i}^{\left(\mathrm{a} 33^{\prime}\right)}$ and $\psi_{i}^{(a, 3)}$ are micro-variables that characterize the linear dependence of the displacement $u_{i}^{(a)}$ on the local coordinates $\bar{x}_{1}^{(a)}, \bar{x}_{2}^{(3)}, \bar{x}_{3}^{(?)}$. In Eq. (A-1) and the sequel, repeated Greek letters do not imply summation. Note that due to the linearity of Eq. (A-1), static equilibrium of the material within the subcell $\left(a 3^{\prime}\right)$ is ensured.

The components of the small strain tensor are given by

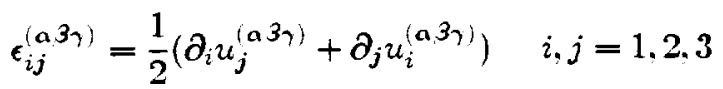

where $\partial_{1}=\partial / \partial \bar{x}_{1}^{(a)}, \partial_{2}=\partial / \partial \bar{x}_{2}^{(3)}$ and $\partial_{3}=\partial / \partial \bar{x}_{3}^{(\gamma)}$.

The volume averaged total strains and stresses in the compasite are expressed, respectively; as

$$
\bar{\epsilon}_{i j}=\frac{1}{d h c} \sum_{a=1}^{N_{a}} \sum_{3=1}^{N_{3}} \sum_{\gamma=1}^{N_{\gamma}} d_{a} h_{3} c_{\gamma} \bar{\epsilon}_{i j}^{\left.(a\}_{\gamma}\right)}
$$

and

$$
\bar{\sigma}_{i j}=\frac{1}{d h c} \sum_{a=1}^{N_{a}} \sum_{3=1}^{N_{3}} \sum_{\gamma=1}^{N_{\gamma}} d_{a} h_{3} h_{\gamma} \bar{\sigma}_{i j}^{\left(a 3_{\gamma}\right)}
$$

with the relationship between the averages of the stress $\bar{\sigma}_{i j}^{\left(a \beta_{\gamma}\right)}$, total strain $\bar{\epsilon}_{i j}^{\left(a \beta_{\gamma}\right)}$, inelastic strain $\bar{\epsilon}_{i j}^{I\left(a \gamma_{\gamma}\right)}$, and thermal strain $\bar{\epsilon}_{i j}^{T(a, 3 \gamma)}=a_{i j}^{(a, 3 \gamma)} \Delta T$ ( where $a_{i j}^{(a, 3 \gamma)}$ is the coefficient of thermal expansion tensor, and $\Delta T$ is the temperature deviation $T-T_{R}$ from a reference temperature $T_{R}$ ) being given by the classic Hookean constitutive equation

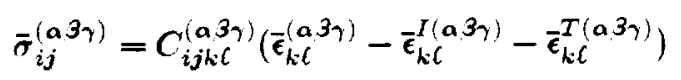

where $C_{i j k \zeta}^{\left(a \zeta^{3}\right)}$ is the elastic stiffiness tensor of the material.

It has been shown ([Paley and Aboudi (1992)] and [Aboudi(1995)]) that by employing the displacement and traction continuity conditions at the interfaces between the subcells of the repeating volume element, and at the interfaces between neighboring repeating volume elements, it is possible to eliminate the microvariables and obtain, via a smoothing operation (homogenization), a set of continuum equations that model the overall behavior of the multiphase short-fiber composite. This was achieved by establishing relationships which connect the microstrains at the subcells to the total overall macrostrains in the composite via the appropriate concentration tensors, $\mathbf{A}$ and $\mathbf{D}$.

\subsection{Overall Thermomechanical Constitutive Law}

Given these concentration matrices, $\mathbf{A}^{\left(a 3_{\gamma}\right)}$ and $\mathbf{D}^{\left(a 3_{\gamma}\right)}$, expressions for the average total strain and stress. respectively, in each suboell can be constructed; that is,

$$
\bar{\epsilon}^{\left(a \beta_{\gamma}\right)}=A^{\left(a \beta_{\gamma}\right)} \bar{\epsilon}+D^{\left(a \gamma_{\gamma}\right)}\left(\epsilon_{s}^{I}+\epsilon_{s}^{T}\right)
$$

and

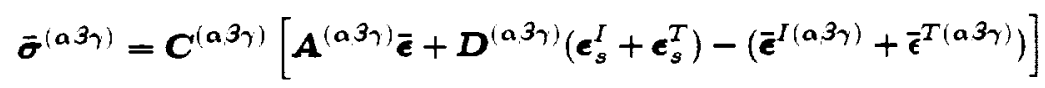


in terms of the uniform overall strain $\bar{\epsilon}$ (the applied macrostrain) and the subcell inelastic and thermal strains, where

$$
\mathbf{A}=\left[\begin{array}{c}
\mathbf{A}_{M} \\
\mathbf{A}_{G}
\end{array}\right]^{-1}\left[\begin{array}{l}
0 \\
\mathbf{J}
\end{array}\right] \quad, \quad \mathbf{D}=\left[\begin{array}{c}
\mathbf{A}_{M} \\
\mathbf{A}_{G}
\end{array}\right]^{-1}\left[\begin{array}{c}
\mathbf{A}_{M} \\
0
\end{array}\right]
$$

and $\mathbf{A}_{M}$ involves the elastic properties $\boldsymbol{C}^{\left(\mathrm{a}^{3}\right)}$ of the subcell material, $\mathbf{A}_{G}$ the geometric dimensions of the repeating cell only; and $\mathbf{J}$ is a matrix containing the impased average (composite) strains. Note, $\left.\mathbf{A}^{\text {(a }} \mathbf{3}^{\prime}\right)$ and $D^{\left(a 3_{9}\right)}$ are square matrices of $6 N_{a} N_{3} N_{\text {; }}$ order. Similarly, notice that the same matrices $D^{\left(a 3_{7}\right)}$ are operating on both the inelastic and thermal strains of the subcells.

It was shown by Dvorak and Benveniste (1992) and Dvorak (1992) that for any representative volume element under a uniform overall strain $\bar{\epsilon}$ and temperature change $\Delta T$, which contains a piecewise uniform distribution of thermal and inelastic fields (eigenstrains) associated with the applied loading $\overline{\boldsymbol{\epsilon}}$, the averages of the local strains can be expressed in terms of the mechanical and eigenstrain concentration tensors. These tensors depend on the local elastic moduli, and on the shape and volume fraction of the phases and are therefore constant. Equation $(A-6)$ is consistent with this representation of the average subcell strain. where the constant mechanical and eigenstrain concentration tensors are given by $\boldsymbol{A}^{\left(a 3^{\prime}\right)}$ and $\boldsymbol{D}^{\left(a 3^{3}\right)}$, respectively. lished

Consequently, the following effective elastoplastic thermomechanical law of the compasite can be estab-

$$
\overline{\boldsymbol{\sigma}}=\boldsymbol{B}^{*}\left(\overline{\boldsymbol{\epsilon}}-\overline{\boldsymbol{\epsilon}}^{I}-\overline{\boldsymbol{\epsilon}}^{T}\right)
$$

where the effective elastic stiffness tensor, $B^{*}$, of the composite is given by

$$
\boldsymbol{B}^{*}=\frac{1}{d h(} \sum_{a=1}^{N_{a}} \sum_{3=1}^{N_{3}} \sum_{\gamma=1}^{N_{\gamma}} d_{a} h_{3} C_{9} \boldsymbol{C}^{\left(a 3_{\gamma}\right)} \boldsymbol{A}^{\left(a 3_{\gamma}\right)}
$$

and the composite inelastic strain tensor is defined as

$$
\overline{\boldsymbol{\epsilon}}^{I}=\frac{-B^{*-1}}{d h \zeta} \sum_{a=1}^{N_{a}} \sum_{\beta=1}^{N_{3}} \sum_{\eta=1}^{N_{\alpha}} d_{a} h_{3} C_{\gamma} \boldsymbol{C}^{\left(a 3_{\gamma}\right)}\left(\boldsymbol{D}^{\left(\mathbf{a} 3_{\gamma}\right)} \boldsymbol{\epsilon}_{s}^{I}-\overline{\boldsymbol{\epsilon}}^{I\left(a 3_{\gamma}\right)}\right)
$$

and the average thermal strain tensor as

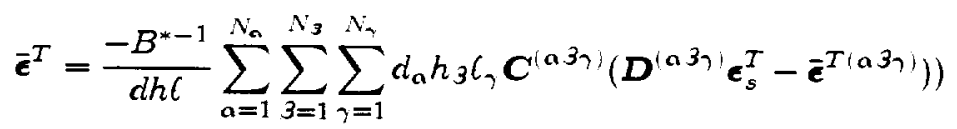

with subcell inelastic, $\epsilon_{s}^{I}$. and thermal, $\epsilon_{s}^{T}$. strains being defined as:

$$
\begin{aligned}
\boldsymbol{\epsilon}_{s}^{I} & =\left(\bar{\epsilon}^{I(111)} \ldots \ldots \overline{\boldsymbol{\epsilon}}^{I\left(N_{a} N_{3} N_{h}\right)}\right) \\
\boldsymbol{\epsilon}_{s}^{T} & =\left(\bar{\epsilon}^{T(111)}, \ldots, \bar{\epsilon}^{T\left(N_{a} N_{3} N_{a}\right)}\right)
\end{aligned}
$$

The effective coefficient of thermal expansion vector, $a^{*}$ of the compasite, is given by

$$
\boldsymbol{\alpha}^{*}=\overline{\boldsymbol{\epsilon}}^{T} / \Delta T
$$

The above micromechanically established overall thermo-inelastic constitutive law (A-S) is valid for any. ty pe of thermomechanical loading (i.e., any combination of normal, shear and thermal loadings). A significant advantage of this constitutive law stems from the fact that it does not rely on any symmetry conditions that may exist under certain types of applied loadings. Thus in the implementation of this law, the question as to whether such symmetry conditions exist or not is irrelevant. 


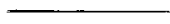$$
\text { 然 }
$$

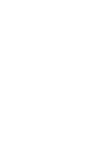

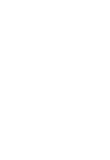

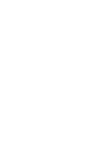

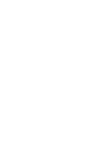

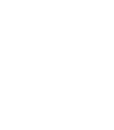

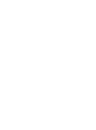




\section{Appendix B: Semi-analytical Axisymmetric Elastoplastic Shear Lag Model}

Given the elastoplastic investigations under taken in this study; a simple shear-lag model for aligned short fiber compasites is introduced. As mentioned in the background section, shear lag models were the first micromechanics models considered for aligned short-fiber composites. Despite some serious theoretical flaws (e.g., the ability to predict only the longitudinal modulus $E_{11}$ within the elastic range). shear lag models have enjoyed enduring popularity; probably due to their algebraic simplicity and their physical appeal. In this paper, we have purposefully limited our investigation to the longitudinal elastoplastic stiffiness beharior so that the propased modified shear lag model can be appropriately utilize.

Following Cox (1952), the shear lag analyses are focussed on a single fiber of length $l_{f}$ and radius $r_{f}$, which is encased in a concentric cylindrical shell of radius $R$. Such a configuration then leads to the key assumption of a shear lag model, which is that the shear stress between fiber and matrix $\left(\tau_{r}, r, \varphi, z \ldots\right.$ cylindrical coordinate system) is proportional to the difference in displacement between the fiber surface and the outer matrix surface, i.e.,

$$
\tau_{r s}(z)=\frac{H}{2 \pi r_{f}}\left(w(R, z)-w\left(r_{f}, z\right)\right)
$$

where $H$ is a constant and can be written as

$$
H=\frac{2 \pi G_{n}}{\ln \left(R / r_{f}\right)-1} .
$$

It remains to choose the radius $R$ of the outer matrix cylinder. Several choices have been used (see e.g. [Tucker and Liang (1999)]). For our purposes it appears most realistic to let $R$ be equal to a radius corresponding to the width of the FE or GNC unit cell. The above equations are based on a one-dimensional analysis of a fiber surrounded by a matrix lajer (i.e., the Poisson effects are neglected (taken to be zero)) and assumes linear elastic material behavior. To extend this analysis into the elastoplastic regime of aligned short fiber composites, the existing shear-lag model must be modified so as to:

1. acoount for elastoplastic material behavior and

2. the appropriate Poisson effect.

The elastoplastic material behavior is simply introduced by replacing $G_{m}$ by the plastic tangent shear modulus $G_{H}$ ("shear hardening slope") and an incremental formulation of Eq. (B-1), i.e.,

$$
d \tau_{r_{i}}(z)=\frac{\bar{H}}{2 \pi r_{f}}\left(d w(R, z)-d w\left(r_{f}, z\right)\right)
$$

with

$$
\bar{H}=\frac{2 \pi G_{H}}{\ln \left(R / r_{f}\right)-1} .
$$

This new "shear hardening slope" can then be obtained from the Prandtl-Reuss-equation. For a pure shear load in the $r$-z-plane this equation can be written as

$$
d \epsilon_{r z}=\frac{1}{2 G_{m}} d \tau_{r z}+\frac{3}{2} \frac{d \tau_{r z}}{\tau_{r z} E_{H}} \tau_{r z}
$$

and leads finally to the shear hardening slope

$$
G_{H}=\frac{d \tau_{r z}}{d \epsilon_{r \varepsilon}}=\psi \frac{G_{m} E_{H}}{E_{H}+3 G_{m}}=\text { const }
$$




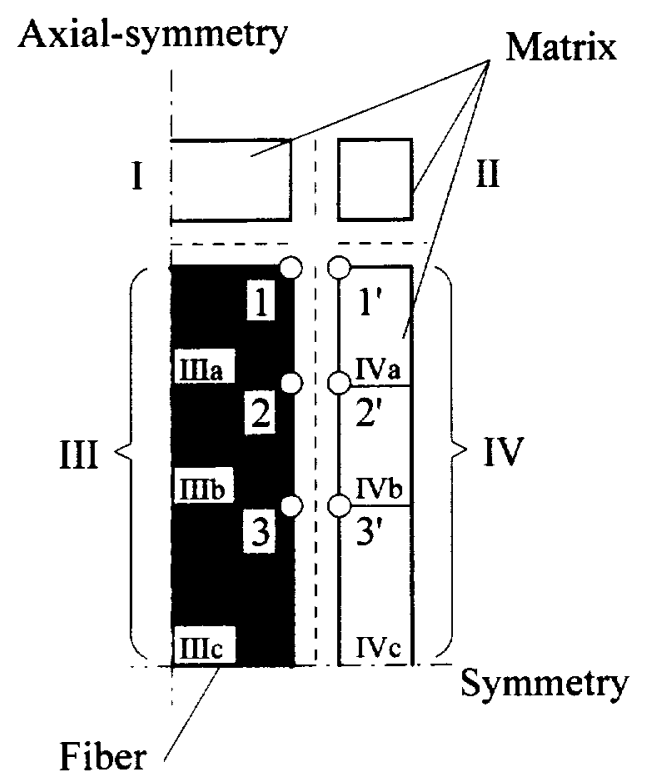

Figure 27: Sketch of the semi-analytical shear lag model

where $G_{H}$ is constant because $E_{H}$ is a constant. Note, within the plastic range a fitting parameter $\psi$ is also introduced. This fitting parameter will acoount for effects which can not be captured by such a simple model.

The Poisson effect is taken into account by considering an axisymmetric model (shown in Fig. 27 using an exploded view) with appropriate boundary conditions on the concentric cylindrical matrix shell.

The model passes the following characteristics:

1. The crass section of the axisymmetric model is divided into four regions $(I, I I, I I I, I V)$. Further, regions $I I I$ and $I V$ are subdivided into three subregions each, e.g., IIIIa, IIIIb, IIIc and $I V a, I V b$, $I V c$ (see Fig. 27).

2. Within all regions only normal stress is allowed (i.e., shear stresses are zero) such that rectangular cross sections of individual regions remain rectangular after loading.

3. The longitudinal stress are constant within regions $I$ and $I I$ and are piecewise constant within regions $I I I$ and $I V$ (i.e., constant within each subregion).

4. The points 1-1', 2-2', etc. are connected with non-linear truss elements which characterize the shear forces between the fiber and the matrix.

5. The stiffiness behavior of each non-linear truss element, $i$, is based on Eq. (B-1), and follows from

$$
F_{\text {thruss }}^{i}=\tau_{r:} 2 \pi r_{f} l_{\tau}^{i}=H\left(w(R, z)-w\left(r_{f}, z\right)\right) l_{\tau}^{i}=C_{\text {truss }}^{i}\left(w(R, z)-w\left(r_{f}, z\right)\right)
$$

where, e.g., for $i=1, l_{\tau}^{1}$ is the distance between points 1 and 2 in Fig. 27 and represents a kind of shear-influence length for truss $1 . C_{t r u s s}^{1}$ represents the stiffness of truss 1 between points 1-1'. The material behavior of the truss element is bilinear and is characterized by a "yield force" (which is based on Tresca's yield condition for pure shear within the interface) and can be written as

$$
F_{t r u s s, Y}^{i}=\frac{\sigma_{Y}}{2} 2 \pi r_{f} l_{\tau}^{i}
$$


Public reporting burden for this collection of information is estimated to average 1 hour per response, including the time for reviewing instructions, searching existing data sources, gathering and maintaining the data needed, and completing and reviewing the collection of information. Send comments regarding this burden estimate or any other aspect of this Davis Highway, Suite 1204, Arlington, VA 22202-4302, and to the Office of Management and Budget, Paperwork Reduction Project (0704-0188), Washington, DC 20503.

\begin{tabular}{|l|l|l}
\hline 1. AGENCY USE ONLY (Leave blank) & $\begin{array}{c}\text { 2. REPOAT DATE } \\
\text { November 2001 }\end{array}$ & $\begin{array}{r}\text { 3. REPORT TYPE AND DATES COVERED } \\
\text { Technical Memorandum }\end{array}$ \\
\hline
\end{tabular}

4. TITLE AND SUBTITLE

The Applicability of the Generalized Method of Cells for Analyzing Discontinuously Reinforced Composites

6. AUTHOR(S)

D.H. Pahr and S.M. Arnold

7. PERFORMING ORGANIZATION NAME(S) AND ADDRESS(ES)

National Aeronautics and Space Administration

John H. Glenn Research Center at Lewis Field

Cleveland, Ohio 44135-3191

9. SPONSORINGMONITORING AGENCY NAME(S) AND ADDRESS(ES)

National Aeronautics and Space Administration

Washington, DC 20546-0001
5. FUNDING NUMBERS

WU-708-72-42-00

B. PERFORMING ORGANIZATION

REPORT NUMBER

E-13026

10. SPONSORINGMONITORING AGENCY REPORT NUMBER

NASA TM-2001-211165

11. SUPPLEMENTARY NOTES

D.H. Pahr, Institute of Lightweight Structures and Aerospace Engineering. Vienna University of Technology, A-1040 Vienna, Austria, and S.M. Arnold, NASA Glenn Research Center. Responsible person, S.M. Arnold, organization code $5920,216-433-3334$.

12a. DISTRIBUTION/AVAILABILITY STATEMENT

12b. DISTRIBUTION CODE

Unclassified - Unlimited

Subject Categories: 24 and 39

Distribution: Nonstandard

Available electronically at http://gltrs.grc,nasa.gov/GLTRS

This publication is available from the NASA Center for AeroSpace Information. 301-621-0390.

13. ABSTRACT (Maximum 200 words)

The paper begins with a short overview of the recent work done in the field of discontinuous reinforced composites, focusing on the different parameters which influence the material behavior of discontinuous reinforced composites, as well as the various analysis approaches undertaken. Based on this overview it became evident, that in order to investigate the enumerated effects in an efficient and comprehensive manner, an alternative approach to the computationally intensive finite-element based micromechanics approach is required. Therefore, an investigation is conducted to demonstrate the utility of utilizing the generalized method of cells (GMC), a semi-analytical micromechanics-based approach, to simulate the elastic and elastoplastic material behavior of aligned short fiber composites. The results are compared with (1) simulations using other micromechanical based mean field models and finite element (FE) unit cell models found in the literature given elastic material behavior, as well as (2) finite element unit cell and a new semianalytical elastoplastic shear lag model in the inelastic range. GMC is shown to definitely have a window of applicability when simulating discontinuously reinforced composite material behavior.

\section{SUBJECT TERMS}

15. NUMBER OF PAGES

38

Micromechanics; Elastic; Plastic; Metal matrix composites; Analysis

19. SECURITY CLASSIFICATION OF ABSTRACT

Unclassified
OF REPORT

Unclassified
18. SECURITY CLASSIFICATION OF THIS PAGE

Unclassified
16. PRICE CODE

20. LIMITATION OF ABSTRACT

tandard Form 298 (Rev. 2-89)

Prescribed by ANSI Std. Z39-18 298-102 
as well as by a stiffness hardening slope which can be written as

$$
C_{\text {truss. } H}^{i}=\psi \bar{H} l_{\tau}^{i} .
$$

The number of introduced truss elements depends on the desired aspect ratio. For $a_{r}=5$ three truss elements were used, whereas for $a_{r}=25$ six trusses were applied.

6. Finally, in addition to the symmetry conditions shown in Fig. 27, symmetry is also required on the top and right outer surface of the axisymmetric model (see Fig. 27).

The introduced semi-analytical shear lag model is solved numerically using the finite element method.

The so called semi-analytical shear-free model is obtained by assuming that the truss elements are characterized by zero stiffness. Such a model represents a standard GMC-model. 